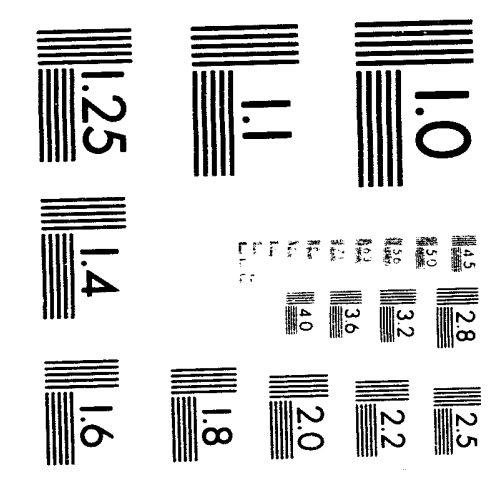



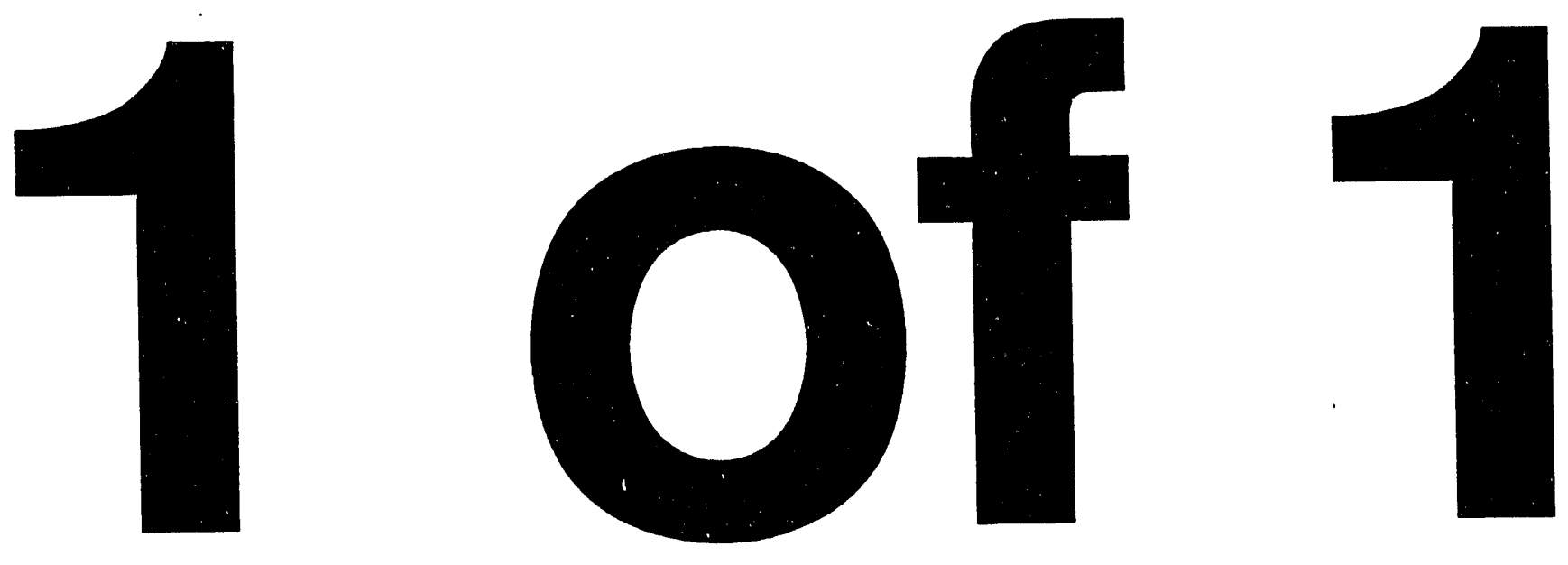
J. H. Shinn

F. J. Gouveia

S. E. Patton

C. O. Fry

April 1993

Prepared for Nevada Field Office, U. S. Department of Energy, Las Vegas, Nevada

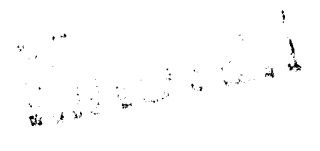


TABLE OF CONTENTS

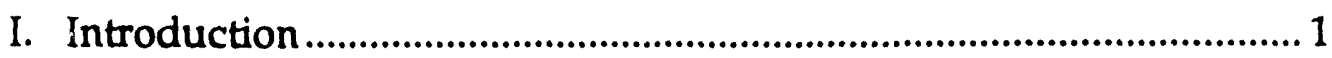

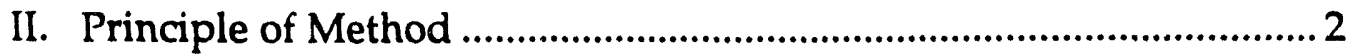

III. Method of Measurement ................................................................... 4

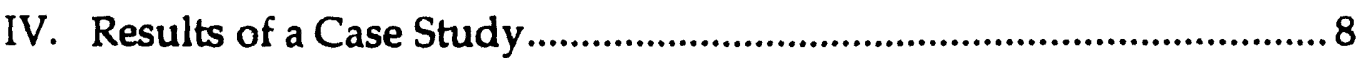

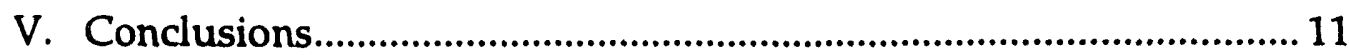

Appendix A: Plots of FIDLER Measurements along Area 11 Transects Appendix B: Area 11 Sampling Locations and FIDLER Measurements Appendix C: Detailed Procedures for the FIDLER 


\title{
AREA 11 CASE STUDY OF RADIONUCLIDE MOVEMENT BY STORM CHANNEL EROSION: A BASELINE METHOD AND INITIAL EVALUATION
}

\author{
J. H. Shinn, F. J. Gouveia, S. E. Patton, and C. O. Fry \\ Lawrence Livermore National Laboratory, Livermore, California
}

\section{INTRODUCTION}

At the Nevada Test Site (NTS), where radionuclide contamination is found in surface soils, there is the potential for water erosion to move radionuclides beyond the boundaries of controlled areas and into channels cut by infrequent storms. This loss of control and the increased risk of further movement are issues which require the development of a method for the quantitative monitoring of contamination and calculation of the radionuclide-movement rate.

The down-slope movement of radionuclides on a surface soil may be so slow as to appear imperceptible, but under the influence of large rainstorms the soil itself moves toward small "washes" and larger water channels. These "storm channels" are the zones of convergence of surface-soil erosion and hence represent the first detectable locations of radionuclide movement by the erosion process. In the arid environment of the NTS, years may pass before a substantial storm calises movement. The stochastic nature of annual rainfall patterns, balance of infiltrationrunoff, and size of the upstream watershed are substantial factors that may contribute to the unpredictable nature of the radionuclide movement.

The perception of radionuclide movement by one "prospecting" with a gamma-ray detector in a storm channel is not sufficient quantification to draw adequate conclusions about the amount and rate of movement. In our past studies at the NTS, we have observed that once the contaminated soil converges into a channel and periodically becomes suspended-sediment, it displays patterns of deposition which demand careful analysis to quantify.

In this report we develop a method which is used to measure the amount and rate of movement of americium-241 $(241 \mathrm{Am})$ in a storm channel, and which offers special features for establishment of baseline concentrations. This method was applied to the standing problem of the erosion of the plutonium contaminated "ground zero" area of site "11D" in Area 11 of NTS. By establishing 241 Am concentrations in the storm channel, the concentrations of $239+240 \mathrm{Pu}$ can also be calculated using a previously determined $239+240 \mathrm{Pu} / 241$ Am ratio from soil samples collected in Area 11. The method utilizes systematic field surveys with a field instrument for detection of low-energy radiation (FIDLER), and provides a computational method, when validated, could become a standard procedure for monitoring radionuclide movement in the washes and storm channels throughout the NTS. 


\section{PRINCIPLE OF METHOD}

The determination of the rate of change of radionuclide concentrations in a storm channel is based on the principle of conservation of mass, which allows a relationship to be developed between time changes in the downstream gradient of the radioactivity and the rate of deposition in the channel. In the process, the total amount of radionuclide present in the channel may also be estimated.

The "velocity" $V(\mathrm{~m} / \mathrm{yr})$ of the radionuclide movement is not a true local transport velocity, but is the result of many small and large storm events (perhaps never observed) over the long term of years of erosion. During the course of longterm sediment transport, a flux deposition, $Q\left(\mathrm{Ci} / \mathrm{m}^{2}\right.$ year), in a layer $h$ containing the sediment, is related to the difference in concentration, $C\left(\mathrm{Ci} / \mathrm{m}^{3}\right)$, between adjacent cross-sections of the wash. Let $d x$ be an element of the downstream distance, $d y$ be an element in the cross-stream section, so that a mass balance may be written as follows:

$Q d x d y=V h d y C$.

Here $C=\left(C_{1}-C_{2}\right)$, where $C_{1}$ is an upstream cross-section concentration and $C_{2}$ is a downstream ciess-section concentration. This is not a model of the physical process causing deposition, but rather is a diagnostic tool for determining the resultant downstream distribution of radionuclides. The application of this principle is to determine, from successive downstream cross-sections, a baseline gradient of radionuclide concentrations. This "baseline" then can be subtracted from the next downstream gradient of radionuclide concentrations measured at a later time in order to calculate a velocity $(V)$.

To do this rigorously, we must take into consideration the cross-stream variation of concentration $(C)$ and to account for any artifact in cross-stream concentration due to the original contaminating event. Let us define a net deposition $q\left(\mathrm{Ci} / \mathrm{m}^{2}\right)$ which can be measured at any point along the cross-stream transect at any fixed time:

$q=h C$.

Over the entire area of study the:e will be a component of radionuclide deposition due to the original contaminating event. We want to remove its influence on the measurement of the movement of radionuclides down the storm channel by defining an original deposition, $q_{0}$, for each transect. For every measurement, $q$, along the cross-stream transect, we will therefore measure an "excess deposition" defined as $q-q_{0}$, and will integrate across the wash at some downstream position, $x$ : 
$I(x)=\int_{-\infty}^{\infty}\left(q-q_{0}\right) d y \quad$ (units: $\left.\mathrm{Ci} / \mathrm{m}\right)$

The integration $I(x)$ will be a negative value when the amount of radionuclides in the wash is less than that on the banks. In this case, the low concentrations may be due to a "scouring" of the wash.

The desired rigor in the characteristic downstream gradient will be obtained by many successive downstream estimates of $I(x)$ followed by an integration of the estimates from some reference point (such as the boundary of the controlled area) to an infinite distance downstream:

$S=\int_{0}^{\infty} I(x) d x \quad$ (units: $\mathrm{Ci}$ ).

Hence, $\mathrm{S}$ is the total deposition in the channel downstream from the reference point due to the sum of all storms, minus the component of deposition due to the original contaminating event. The characteristic distance of the transport, $L$, is given by $S$ divided by the value of $I(x)$ at the reference point, $I(0)$ :

$L=S / I(0) \quad$ (units: $m$ ).

For example, if the function $I(x)$ is exponential and decreases to a value $1 / \mathrm{e}$ at distance $L$, then the integral $S=L I(0)$. The change in the characteristic baseline integral over time $t$ can be used to calculate the velocity $V$, and in the case of the first baseline, it can be assumed that there was no initial excess deposition in the storm channel:

$V=L / t \quad$ (units: $\mathrm{m} / \mathrm{y}$ )

We will use the FIDLER to measure the approximate value of $q$ across the wash because we expect it to measure $q$ directly without some special knowledge of $h$, although this approximation requires some justification. Furthermore, the FIDLER can be held at a short distance above the soil to measure $q$ with a narrow field of view so that detail can be preserved in $I(x)$.

The FIDLER field of view and the approximate calibration are subjects of consideration. Although the function describing the FIDLER response field of view (FOV) is known exactly, as a rule of thumb using $H$ as the detector height, the FOV measured from a point directly below is $50 \%$ at a distance $1.2 \mathrm{H}, 80 \%$ at a distance $2.5 \mathrm{H}$, and $90 \%$ at a distance $4 \mathrm{H}$. The standard height used at NTS is $1 \mathrm{ft}(0.30 \mathrm{~m})$, so we recommend that $q$ be measured at distances separated every $2.5 \mathrm{ft}(0.75 \mathrm{~m})$ across a wash to have a $80 \%$ response and at the same time maximize the detail obtained by using a small spacing. 
The FIDLER calibration relating net counts-per-minute (cpm) to the net deposition, $q$, will depend in part, on the vertical variation of radionuclides in the soil. In the case of radionuclides deposited by infrequently-suspended sediments, there is no practical method of knowing the vertical distribution, which could vary greatly across the channel. This is not a major limitation, however, to the FIDLER application if the following arguments are accepted. First, a mean value for the calibration will be acceptable because as the FIDLER is moved across many points along a cross-section and at many such cross-sections, there should be no systematic bias in any direction. This means that characteristic gradients so measured will not have any bias, and that the length $L$ and velocity $V$ estimates will also be unbiased. In other words, any calibration bias cancels with the ratio $I(x) / I(0)$. Second, if we are willing to accept a factor of two error of estimation in the total deposition $S$, we can find an approximate mean calibration value from the known FIDLER response. That is, if the vertical distribution of radionuclides in the wash sediments is either an exponential distribution with relaxation depth anywhere between 0.1 and $5 \mathrm{~cm}$, or is a uniform distribution with depth anywhere between 2 and $7.5 \mathrm{~cm}$, then it can be shown by the known FIDLER response that a mean calibration for a relaxation depth of $1.43 \mathrm{~cm}$ will cause less than a factor of two in error of estimation of total deposition $S$. This error is acceptable if we take precautions with the value so estimated; for example, it is only to decide whether an alarming amount of radionuclide tias moved.

On the other hand, the baseline $L$ established by integration has no such limitation, and should disclose movement in subsequent surveys. Since $I(x) / I(0)$ determined by the FIDLER has no strong dependency upon calibration, the value of $L$, which represents the characteristic distance of radionuclide movement downstream, can be obtained at any time in the future to determine if there has been change. The integral value of $L$ is much more robust than other alternatives, such as determining the location of some particular limiting value of the deposition $q$ or the concentration $C$.

\section{METHOD OF MEASUREMENT}

A FIDLER was used for the field measurement of $241 \mathrm{Am}$ in the soils and wash of site 11D. Specific procedures were used for the set-up and calibration of the FIDLER, taking of field measurements, and data analysis. These procedures were prepared as part of the DOE/Nevada Field Office (NV) Basic Environmental Compliance and Monitoring Program (BECAMP) Quality-Assurance Program. The procedures were the "BECAMP Quality Assurance Detailed Procedure for FIDLER Set-up and Calibration" (BECAMP-DP-401,R0), "BECAMP Quality Assurance Detailed Procedure for In Situ Measurements" (BECAMP-DP-402,R0), and "BECAMP Qyality Assurance Detailed Procedure for FILDER Data Analysis" (BECAMP-DP$403, R 0)$. Copies of these procedures can be found in Appendix C. 
A Bicron FIDLER probe and Eberline ESP-2 Smart Portable survey instrument were set-up and calibrated for the detection of $60 \mathrm{keV}$ gamma rays from ${ }^{241} \mathrm{Am}$. This procedure (BECAMP-DP-401,R0) involved setting the ESP-2 analyzer high voltage and gain, determining a 50\% window for the threshold voltage, and determining the FIDLER operating voltage. After set-up and calibration, the counting efficiency of the FIDLER was determined. The counting efficiency represents the number of photopeak counts recorded by the FIDLER per photon per $\mathrm{cm}^{2}$ of detector face cross-section, when an ${ }^{241}$ Am point source is placed at a known height under the detector.

In the field, procedures for measuring ${ }^{241} \mathrm{Am}$ activity involved placing the FIDLER on a tripod stand, which situated the face of the detector one foot $(30 \mathrm{~cm})$ above the ground, and taking one-minute counts (BECAMP-DP-402,R0). The counts were recorded both in a field notebook and in the memory of the ESP-2 analyzer. The general background ${ }^{241} \mathrm{Am}$ activity for Area 11 was determined by taking two one-minute counts at a specific point outside the main gate to Area 11, at a site representative of the geographic condition of the area. These measurements were used for a field check of the FIDLER efficiency several times during the period of field measurements. At site 11D, one-minute counts of ${ }^{241} \mathrm{Am}$ activity were taken at points along the presurveyed transects that crossed the 11D wash. After measurements were made, the data in the ESP-2 analyzer was electronically transferred to a computer database. The database was then verified by comparison with the entries in the field notebook before analysis of the data was begun.

From the calibration of the FIDLER, in situ measurements of ${ }^{241}$ Am activity can be quantified (BECAMP-DP-403,R0). A computer program provides factors based on the FIDLER counting efficiency and angular response measurements, when multiplied by FIDLER in situ measurements, will give soil radionuclide concentrations.

Measurements of $241 \mathrm{Am}$ activity were made with a FIDLER at points along thirteen transects established across two small washes which converge into a single storm channel southeast of the permanent fence around site 11D in Area 11 of the NTS (Figure 1). Measurements were made at $2.5-\mathrm{ft}(0.76 \mathrm{~m})$ intervals along each transect. These transects were not perfectly orthogonal to the main direction of the channel, but were chosen to coincide with a grid previously established by the DOE/NV Nevada Applied Ecology Group (NAEG). Nine transects of the washcomplex are on NAEG 25 -foot grid lines $(7.6 \mathrm{~m})$ numbered $29,30,31,32,33,34,35$, 36 , and 38 . Those nine transects were made in the block between north-south rows lettered $B$ to $N$. Three additional transects were located outside that block and were marked by positions lettered $T, U$, and $V$. Benchmark NTS coordinates for four points in the block and points $T, U$, and $V$ are given in Table 1 . The twelve transects of the washes and stream channel were made very nearly along the direction of 


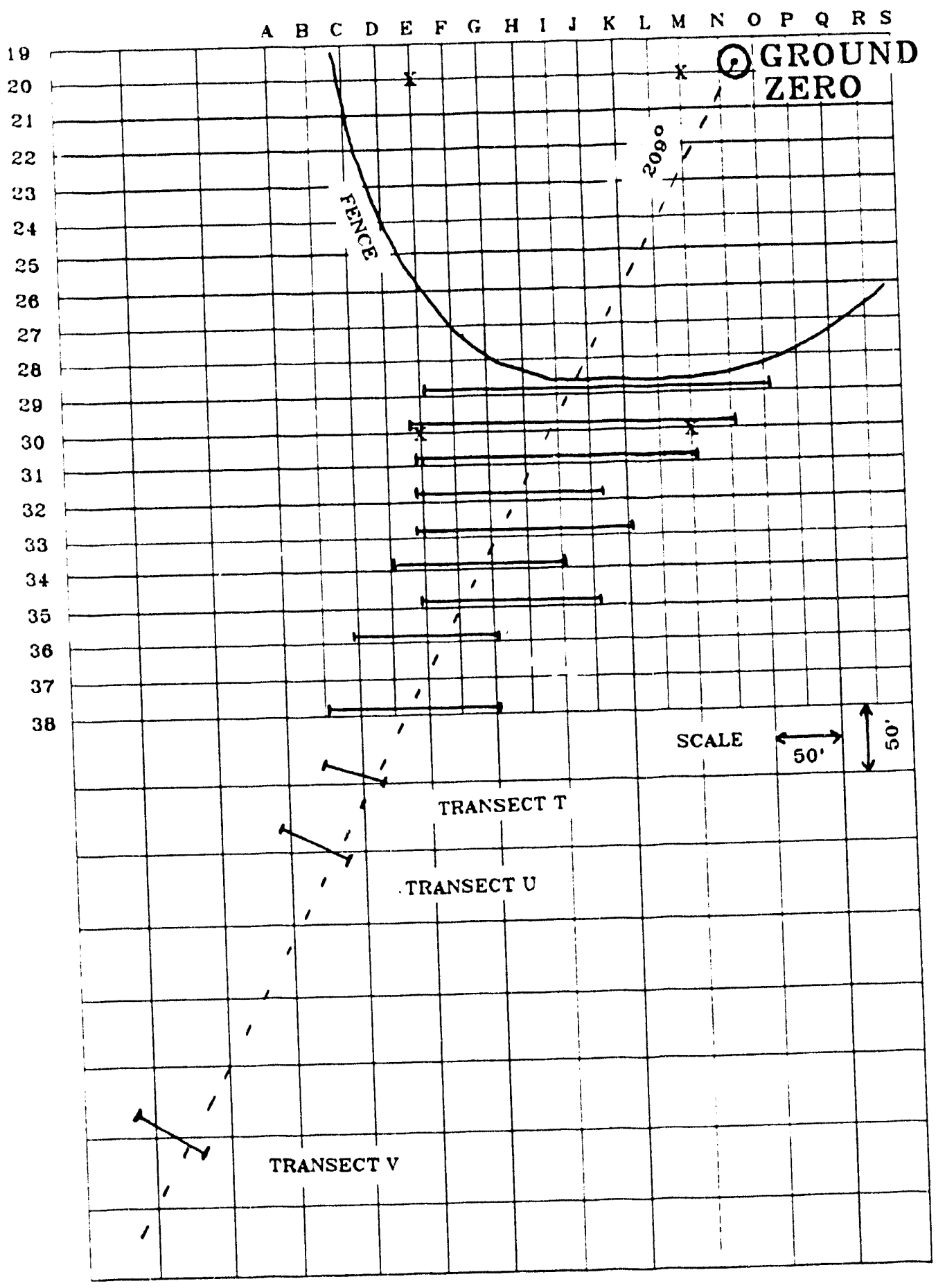

Figure 1. Location of twelve transects south of the ground zero, at site D in Area 11 of the Nevada Test Site, where measurements were made for determination of $241 \mathrm{Am}$ concentrations in the storm channel. The four points on the figure marked with an " $X$ " represent the locations of NTS grid coordinates referenced in Table 1. 
209-degrees downstream from the ground zero, at positions between $250 \mathrm{ft}(76 \mathrm{~m})$ at transect 29 and $865 \mathrm{ft}(263 \mathrm{~m})$ at transect $V$ from the ground zero. Transect 29 is adjacent to the perimeter fence and was the location of the benchmark reference integral I(0). Thus, we were able to make estimates of 241 Am transport "outside" the fence. Not shown on Figure 1 is the location of a thirteenth transect which was made across the storm channel over $2000 \mathrm{~m}$ from the ground zero. The transect was established $10 \mathrm{~m}$ west of the Area 11 access road. No NTS grid coordinates are available. This transect, labeled transect $Z$, was established as a reference baseline measurement of $241 \mathrm{Am}$ activity for monitoring radionculide movment near the of radionuclides from Area 11.

Table 1. Benchmark NTs Coordinates, Site 11D.

\begin{tabular}{rc}
\hline NAEG Reference & NTS Point Coordinates \\
\hline E20 & N809325, E707800 \\
M20 & N809325, E708000 \\
E30 & N809075, E707800 \\
M30 & N809075, E708000 \\
T & N808825, E707760 \\
U & N808775, E707740 \\
V & N808565, E707629 \\
\hline
\end{tabular}

A classification system was used at each 2.5 -foot $(0.76 \mathrm{~m})$ grid point to represent the condition of the surface. This classification was the standard system for all FIDLER surveys conducted by the DOE/NV BECAMP program, except we added one additional classification (No. 7), where it was observed that the storm channel had on occasion "overflowed" its banks, presumably then transporting radionuclides and depositing on top of the desert pavement. The classification system is given in Table 2.

Table 2. Soil surface classification codes.

\begin{tabular}{cl}
\hline Code & \multicolumn{1}{c}{ Description } \\
\hline 1 & Desert Pavement \\
2 & Blow-sand Mound \\
3 & Between Bushes \\
4 & Sand and Gravel \\
5 & Wash Area \\
6 & Rodent Burrows \\
7 & Wash Overflow Area \\
\hline
\end{tabular}


A set of criteria was used in selecting FIDLER measurements as activity due to original deposition from the event $\left(q_{0}\right)$. First, the points of the transect where measurements were made had to be out of the storm channel and not affected by storm events, i.e. overflow areas or areas scoured by runoff. These points would correspond to a soil surface classification of $1,2,3$, or 4 (see Table 2). Second, on inspection of the data, the lower values for these points were used even if they were not the lowest values along the transect. Third, for each transect a minimum of two values were used in determining $q_{0}$, with no maximum to the number of values that could be used.

\section{RESULTS OF A CASE STUDY}

Measurements of the $241 \mathrm{Am}$ deposition in a storm channel downstream of the ground zero at site 11D (Area 11, Nevada Test Site) were made on November 19, 20, and 21, December 12, and December 18, 1991. The FIDLER was used to measure 241 Am activity in the washes and the efficiency of the FIDLER, checked in the field with a certified calibration source, had a mean of $79 \%$ with a standard deviation (S.D.) of $1.9 \%$. The field measurements showed that the majority of the radionuclide deposition was found adjacent to either stream bank. In the center of the stream channel, a "clean" area scoured by the erosional transport during storm runoff was likely to be found. The overflow areas of the stream channel often showed elevated deposition compared to the local background on the desert pavement. The measurements made with the FIDLER (in counts-per-minute) versus cross-section position in the transect, for each transect, are presented in Appendix $A$, Figures A1 through A13. In the figures, numbers printed on the plots show the Table 2 soil surface classifications at each sampling point along the transect and the circled soil surface classifications on the plots were the points used in establishing 9o. A listing of the FIDLER in situ measurements can be found in Appendix B.

For each transect, the $y$-integral value $I(x)$ was computed from the area under the curve using the Simpson's rule method, first subtracting off the estimated background $\left(q_{0}\right)$. The integral values are given in Table 3 for each transect along with the approximate distance of the transect downstream from the ground zero at site 11D. The units of $I(x)$ are given in counts-per-minute per meter (cpm-m) as computed directly from the raw data. To convert these data to the approximate integrated deposition $(\mu \mathrm{Ci} / \mathrm{m})$, we divided by the average FIDLER calibration factor. The calibration factor, based on the set-up and calibration of the FIDLER, was determined have a value of $2120 \mathrm{cpm}$ per $\mu \mathrm{Ci} / \mathrm{m}^{2}$ for ${ }^{241} \mathrm{Am}$. For example, at the transect near the fence (transect 29$), I(x)$ had a value of $1.07 \times 10^{6} \mathrm{cpm}-\mathrm{m}$ which is equivalent to $505 \mu \mathrm{Ci} / \mathrm{m}$. 
Table 3. Cross-section Integrals $I(x)$ of 241 Am Concentrations at Downstream Distances from the Ground Zero at Site 11D.

\begin{tabular}{cccc}
\hline Transect & $\begin{array}{c}\text { Distance } \\
(\mathrm{m})\end{array}$ & $\begin{array}{c}I(x) \\
\left(10^{3} \mathrm{cpm}-\mathrm{m}\right)\end{array}$ & $\begin{array}{c}I(x) \\
(\mu \mathrm{Ci} / \mathrm{m})\end{array}$ \\
\hline 29 & 79 & 1150 & 544 \\
30 & 88 & 891 & 420 \\
31 & 97 & 1110 & 524 \\
32 & 106 & 293 & 138 \\
33 & 115 & 222 & 105 \\
34 & 124 & 284 & 134 \\
35 & 133 & 284 & 134 \\
36 & 141 & 181 & 86 \\
38 & 159 & 137 & 64 \\
$\mathrm{~T}$ & 175 & 39 & 19 \\
$\mathrm{U}$ & 191 & 57 & 27 \\
$\mathrm{~V}$ & 264 & 49 & 23 \\
$\mathrm{Z}$ & $2000+$ & 1.6 & 0.8 \\
\hline
\end{tabular}

The downstream variation of $I(x)$ was approximately exponential for distances from the fence perimeter (transect 29) to about $190 \mathrm{~m}$ downstream (transect $U$ ), as shown in Figure 2. Transects $V$ and $A$ are were considered to far down the stream to be considered in the analysis. The characteristic transport distance, $L$, was then determined by least-squares analysis of the first 11 transects from the fence (transect 29). This analysis gave an estimate of $L$ of $34 \mathrm{~m}$ with a standard error of $3.5 \mathrm{~m}$. The intercept $I(0)$ was estimated to be $505 \mu \mathrm{Ci} / \mathrm{m}$ in this analysis. The elapsed time since the original contaminating events until these measurements $(t)$ is $31 \mathrm{y} \pm 3 \mathrm{y}$ because the events took place over an extended time. Thus, the estimated effective transport velocity, $V$, is about $1 \mathrm{~m} / \mathrm{y}$.

The estimated integrated deposition in the storm channel uses $I(0)$ of $505 \mu \mathrm{Ci} / \mathrm{m}$, estimated above, to calculate $S=L I(0)$ for ${ }^{241} \mathrm{Am}$ in the storm channel. For a $L$ of $34 \mathrm{~m}$ and $I(0)$ of $505 \mu \mathrm{Ci} / \mathrm{m}, S=17.4 \times 10^{3} \mu \mathrm{Ci}$. Since the ratio of $239+240 \mathrm{Pu} / 241 \mathrm{Am}$ in Area 11 is about 6, the amount of $239+240 \mathrm{Pu}$ was estimated to be approximately $104 \times 10^{3} \mu \mathrm{Ci}$. These estimated values for $241 \mathrm{Am}$ and $239+240 \mathrm{Pu}$ are a very small fraction of the total inventory (less than $1 / 10,000$ ) within the site 11D fence. But these values are uncertain by a factor of two as previously discussed in section II, Principle of Method. Hence, we found that the amount of $241 \mathrm{Am}$ in the wash was between 8.4 and $35 \mathrm{mCi}$. Similarly, the S-value of $239+240 \mathrm{Pu}$ would range between 50 and $210 \mathrm{mCi}$. 


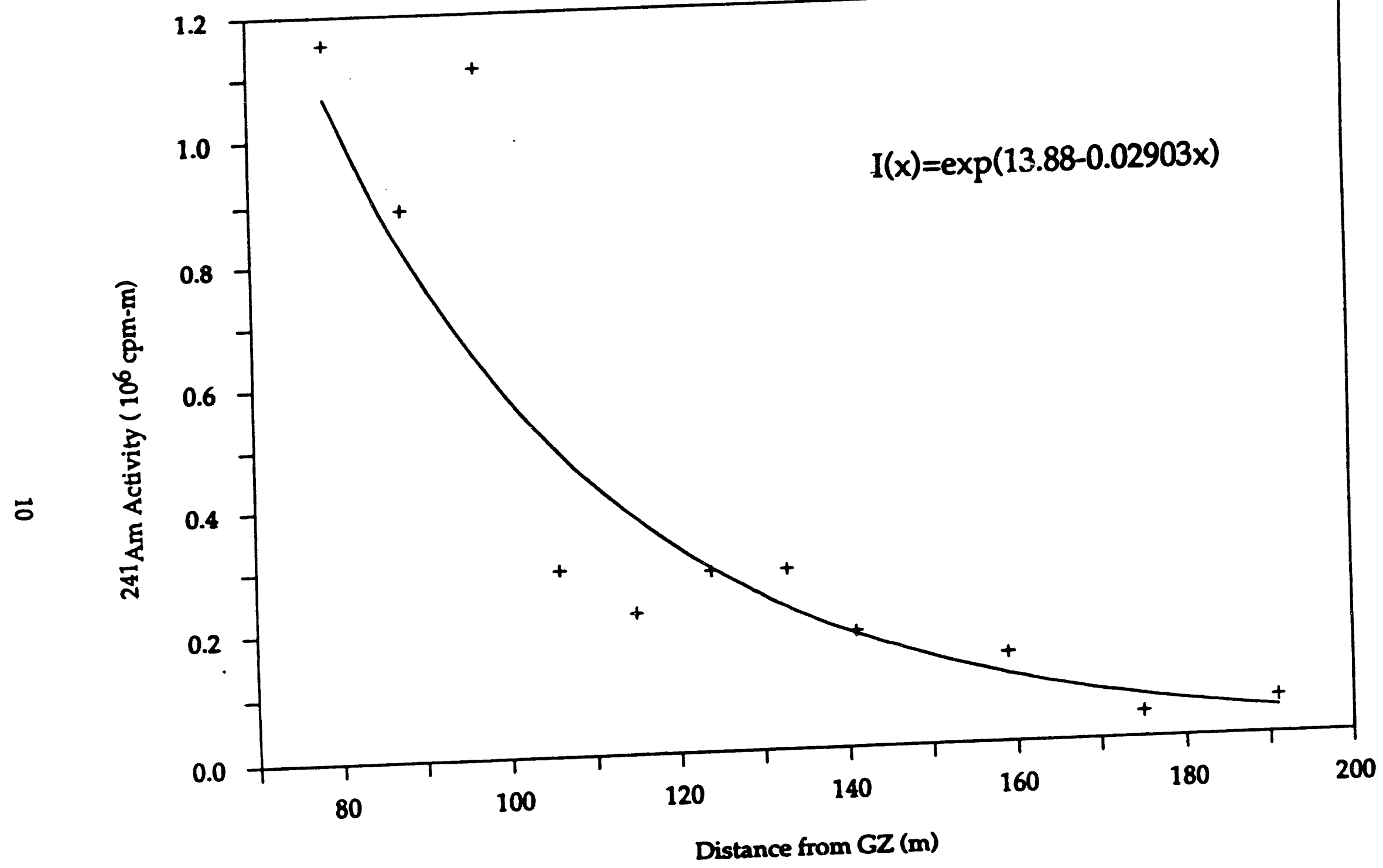

Figure 2. Plot of the cross section integrals $I(x)$ of 241 Am activity at downstream distances from the ground zero at site 11D in Area 11 of the Nevada Test Site. The value of $x$ is taken to be zero at the fence perimeter $(70 \mathrm{~m})$. 
Where possible, we compared our results to quality-control data within our own study, and to other surveys conducted in Area 11. For 25 duplicate measurements made with the FIDLER, the mean relative difference was $5.8 \%$ with a standard deviation of $6.7 \%$, which assures us that our field survey was at least internally consistent. In addition, we measured ${ }^{241} \mathrm{Am}$ at a number of grid point locations which were in common with another survey previously conducted by the DOE/NV Radionuclide Inventory and Distribution Program (RIDP) in Area 11, outside the zone of the wash. Comparison of our data with ranges published by RIDP shows that a high \% of our measurements are within a factor of two (Table 4).

Table 4. Comparison of FIDLER ${ }^{241}$ Am Measurements to 241 Am Ranges Measured by RIDP for the Same Locations.

\begin{tabular}{ccccc}
\hline $\begin{array}{c}\text { RIDP } \\
\text { Stake }\end{array}$ & $\begin{array}{c}\text { RIDP Soil } \\
\text { Conc. } \\
\left(\mu \mathrm{Ci} / \mathrm{m}^{2}\right)\end{array}$ & $\begin{array}{c}\text { RIDP In Situ } \\
\text { Min. } \\
\left(\mu \mathrm{Ci} / \mathrm{m}^{2}\right)\end{array}$ & $\begin{array}{c}\text { Case Study } \\
\text { Survey } \\
\left(\mu \mathrm{Ci} / \mathrm{m}^{2}\right)\end{array}$ \\
\hline 90 & 6.0 & 3.0 & 5.0 & 5.0 \\
95 & 4.6 & 5.0 & 6.0 & 4.0 \\
97 & 9.7 & 9.7 & 10.7 & 7.2 \\
\hline
\end{tabular}

\section{CONCLUSIONS}

A relatively simple method has been demonstrated for monitoring the radionuclide movement by storm channel erosion. The method utilizes a robust integral iechnique to establish the characteristic distance of sediment transport by storms, and an approximate estimate of the total deposition of radionuclides in the sediment.

A case study of the wash at site 11D, Area 11, Nevada Test Site, showed that after about 31 years the transport distance was about $32 \mathrm{~m}$ for an average velocity of $1 \mathrm{~m} / \mathrm{y}$. With data from 12 transects of the wash, it was estimated that about 8.4 to $35 \mathrm{mCi}$ of $241 \mathrm{Am}$ and 50 to $210 \mathrm{mCi}$ of $239+240 \mathrm{Pu}$ had been transported outside the perimeter of the fenced zone around the ground zero. This was a very small fraction (less than $1 / 10,000$ ) of those radionuclides within the fence. 
APPENDIX A: PLOTS OF FIDLER MEASUREMENTS ALONG AREA 11 TRANSECTS 


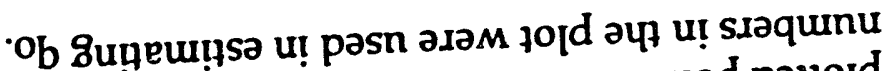

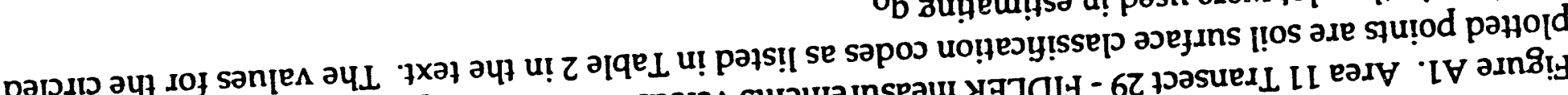
әчұ

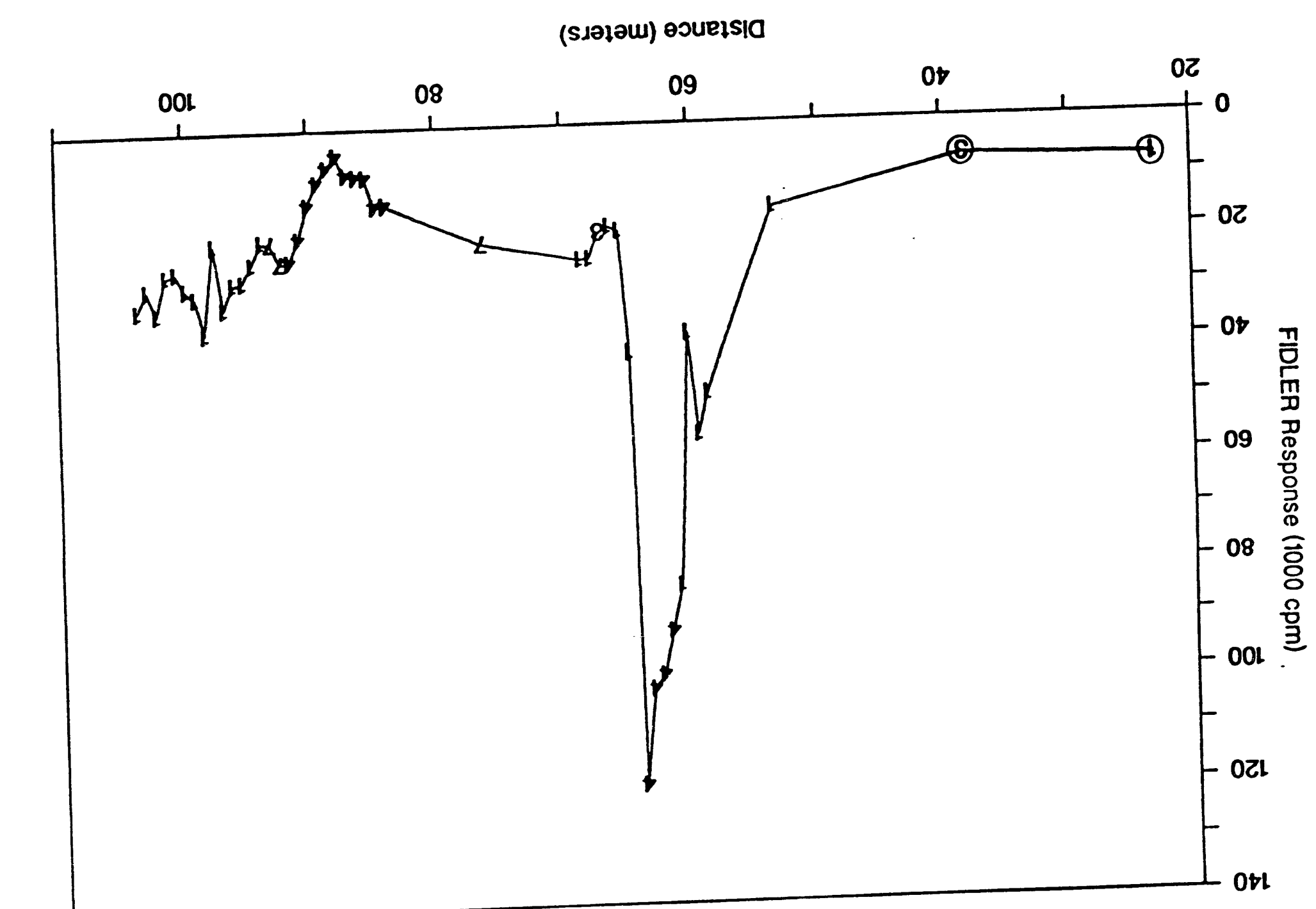




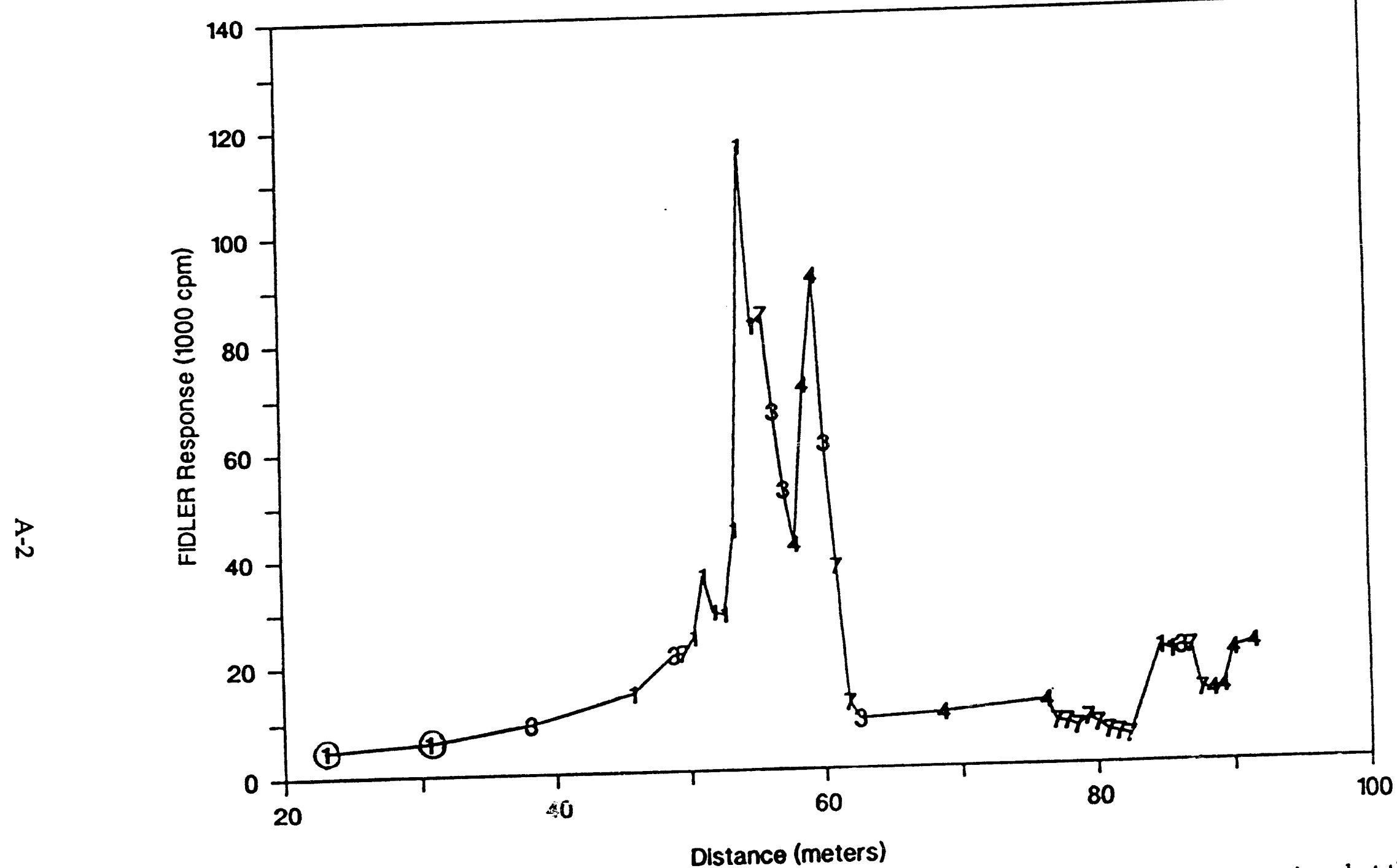

Figure A2. Area 11 Transect 30 - FIDLER measurements versus cross-stream position. The numbers printed at the classification codes as listed in Table 2 in the text. The values for the circled plotted points are soil surface classintimating $q_{0}$. 


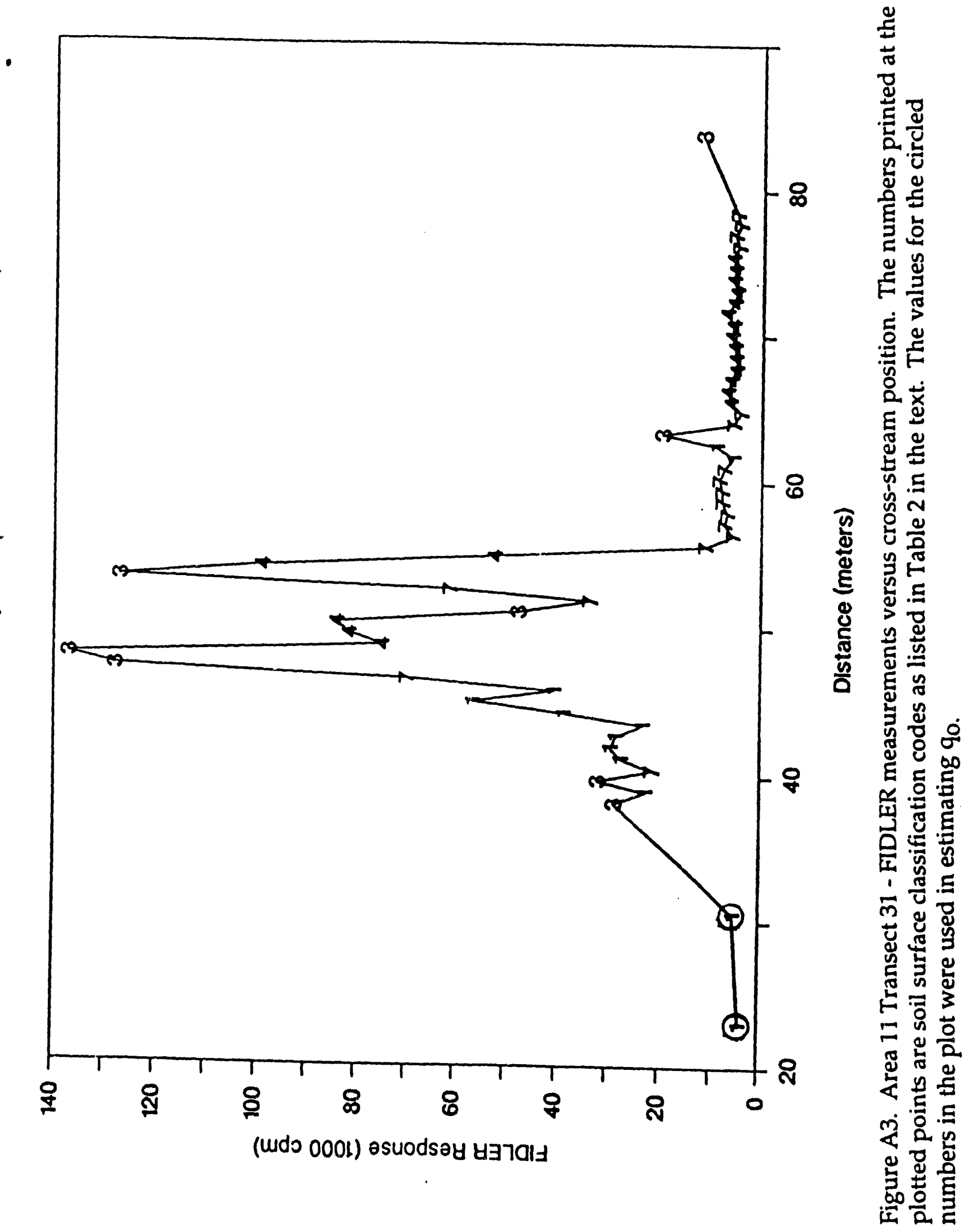




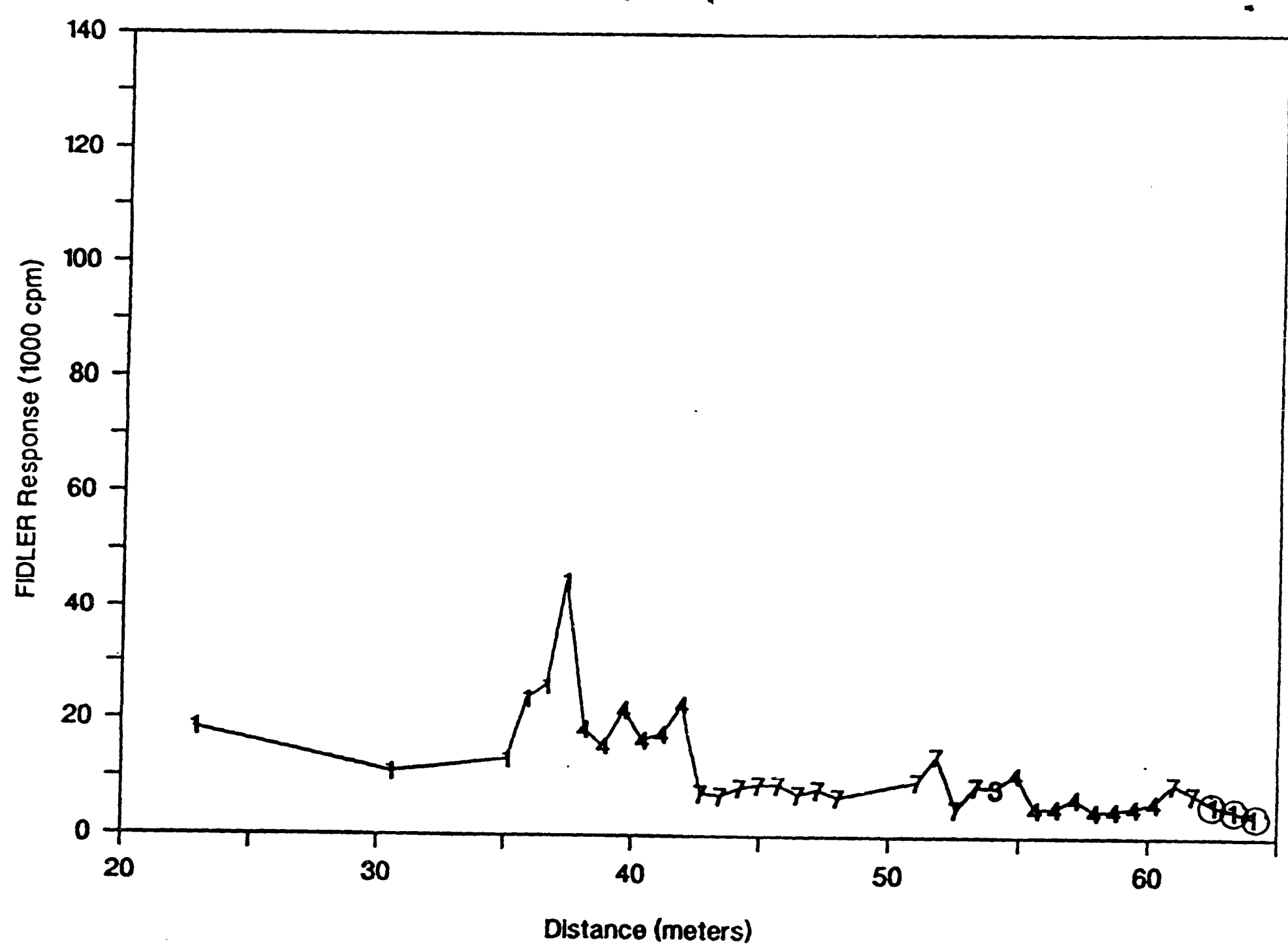

Figure A4. Area 11 Transect 32 - FIDLER measurements versus cross-stream position. The numbers printed at the plotted points are soil surface classification codes as listed in Table 2 in the text. The values for the circled numbers in the plot were used in estimating $q_{0}$. 
ob gupeut!sa u! pasn aram łold aut u! sraqunu

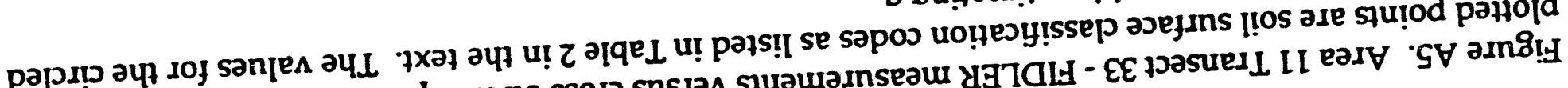

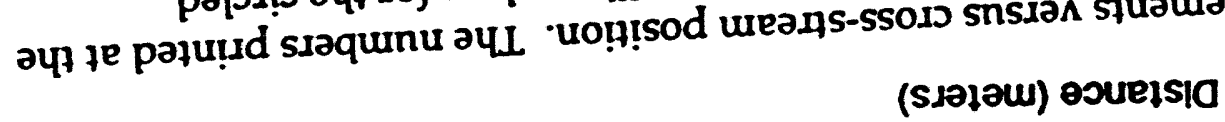

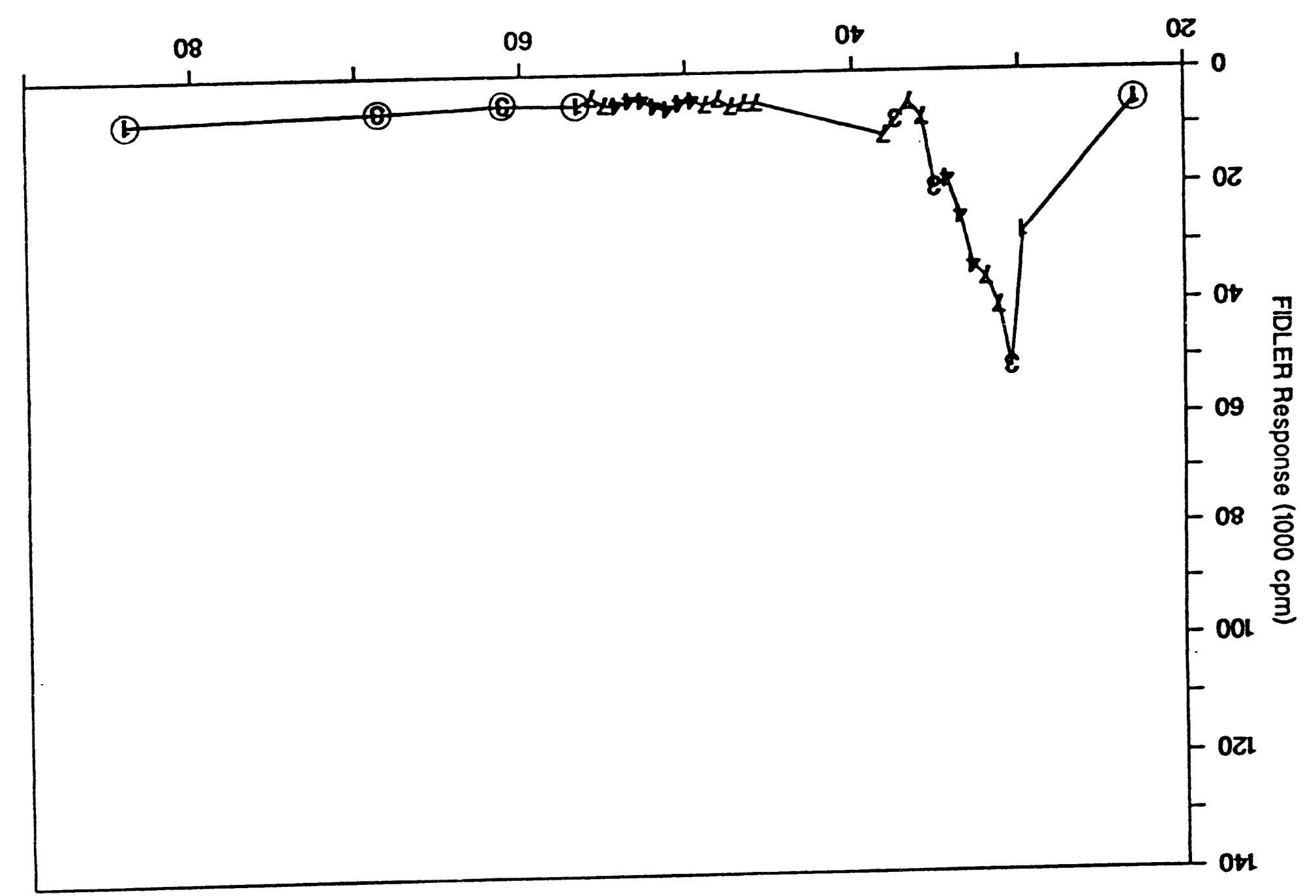




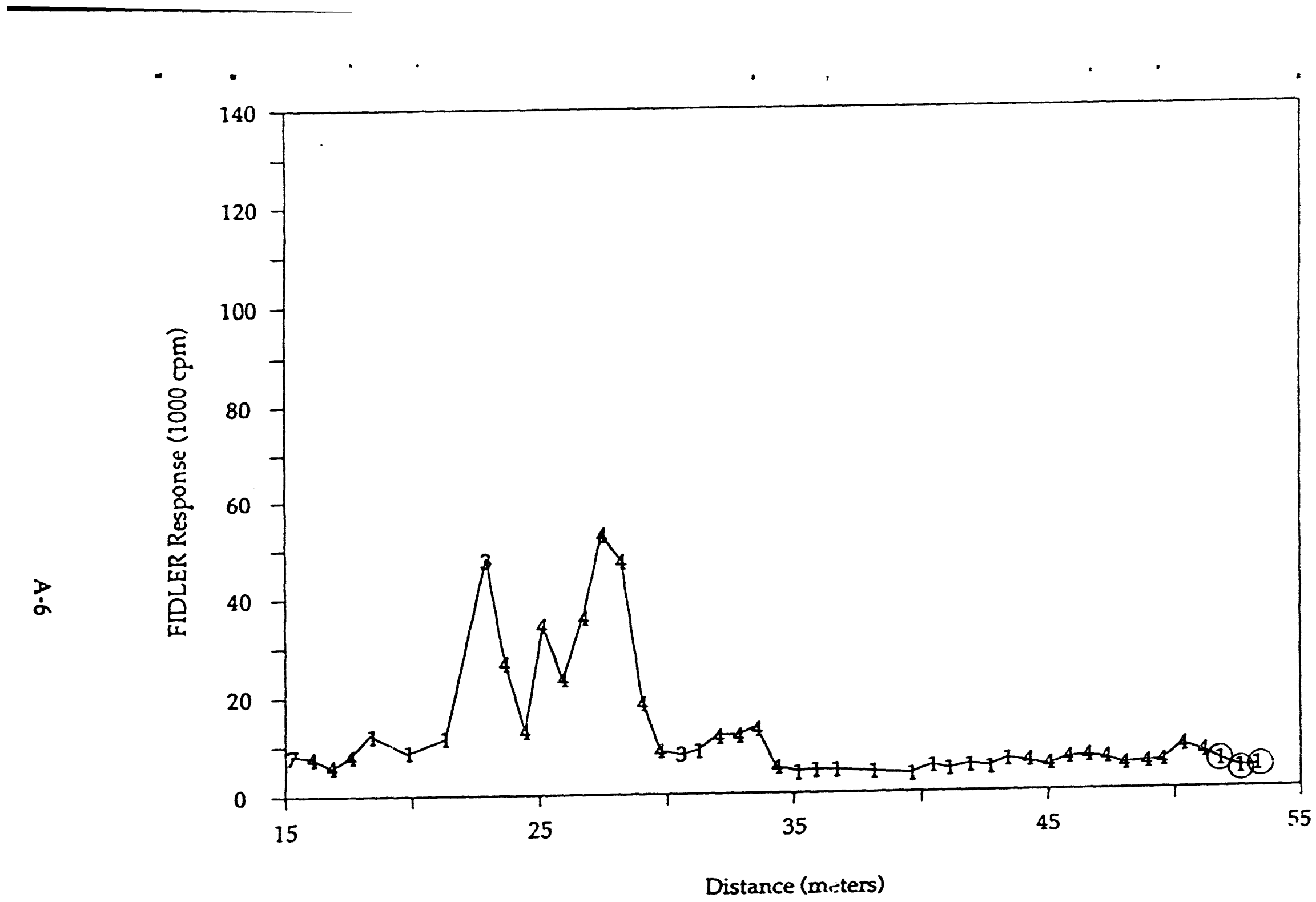

34 - FIDLER measurements versus cross-stream position. The numbers printed at the Figure A6. Area 11 Transect 34 - FIDLER measurements versus cross-stream pos the circled plotted points are soil surface classification codes
numbers in the plot were used in estimating $9_{0}$. 


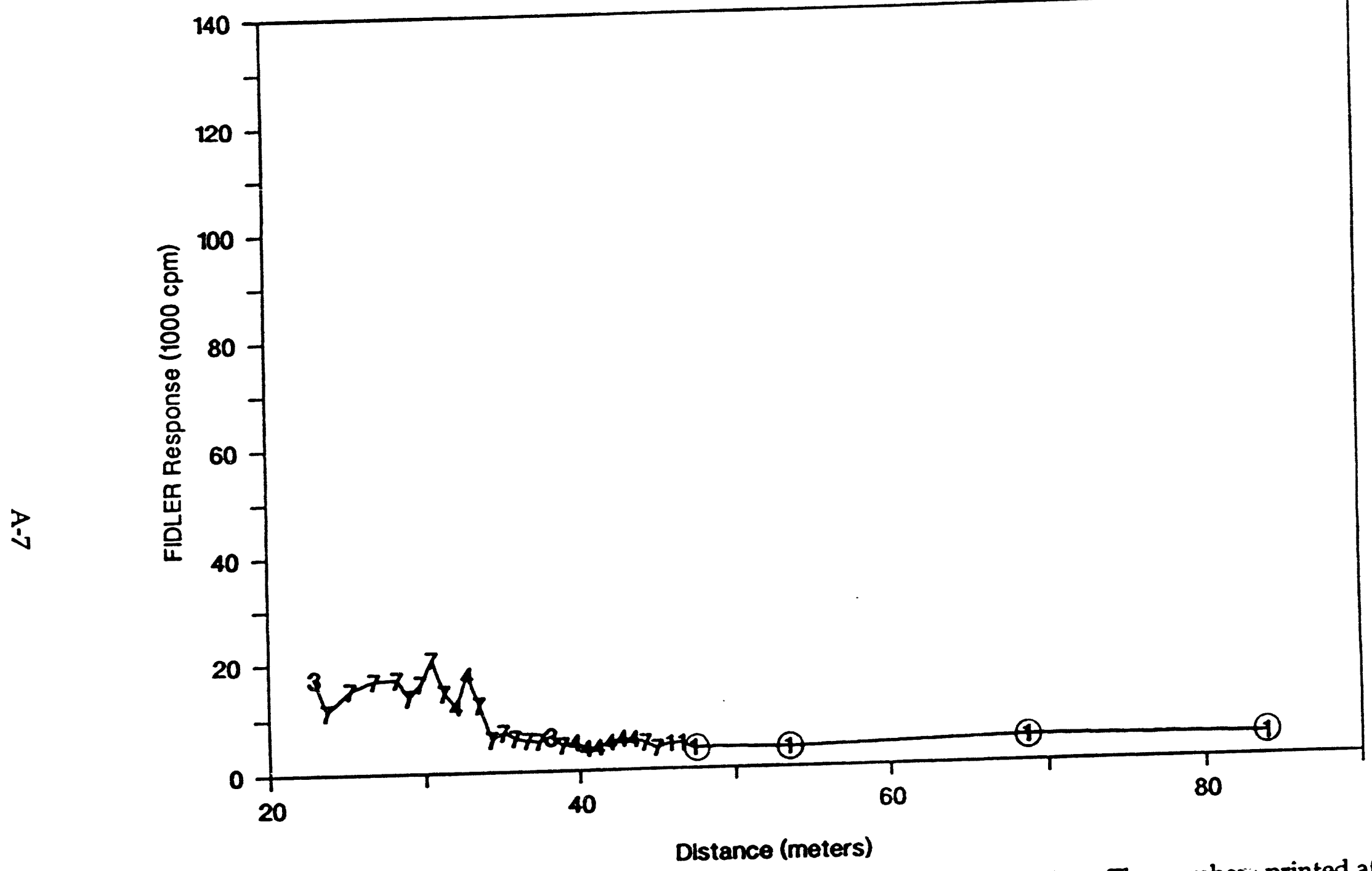

Figure A7. Area 11 Transect 35 - FIDLER measurements versus cross-stream position. The numbers printed at the plotted points are soil surface classification codes as listed in Table 2 in the text. The values for the circled numbers in the plot were used in estimating $q_{0}$. 


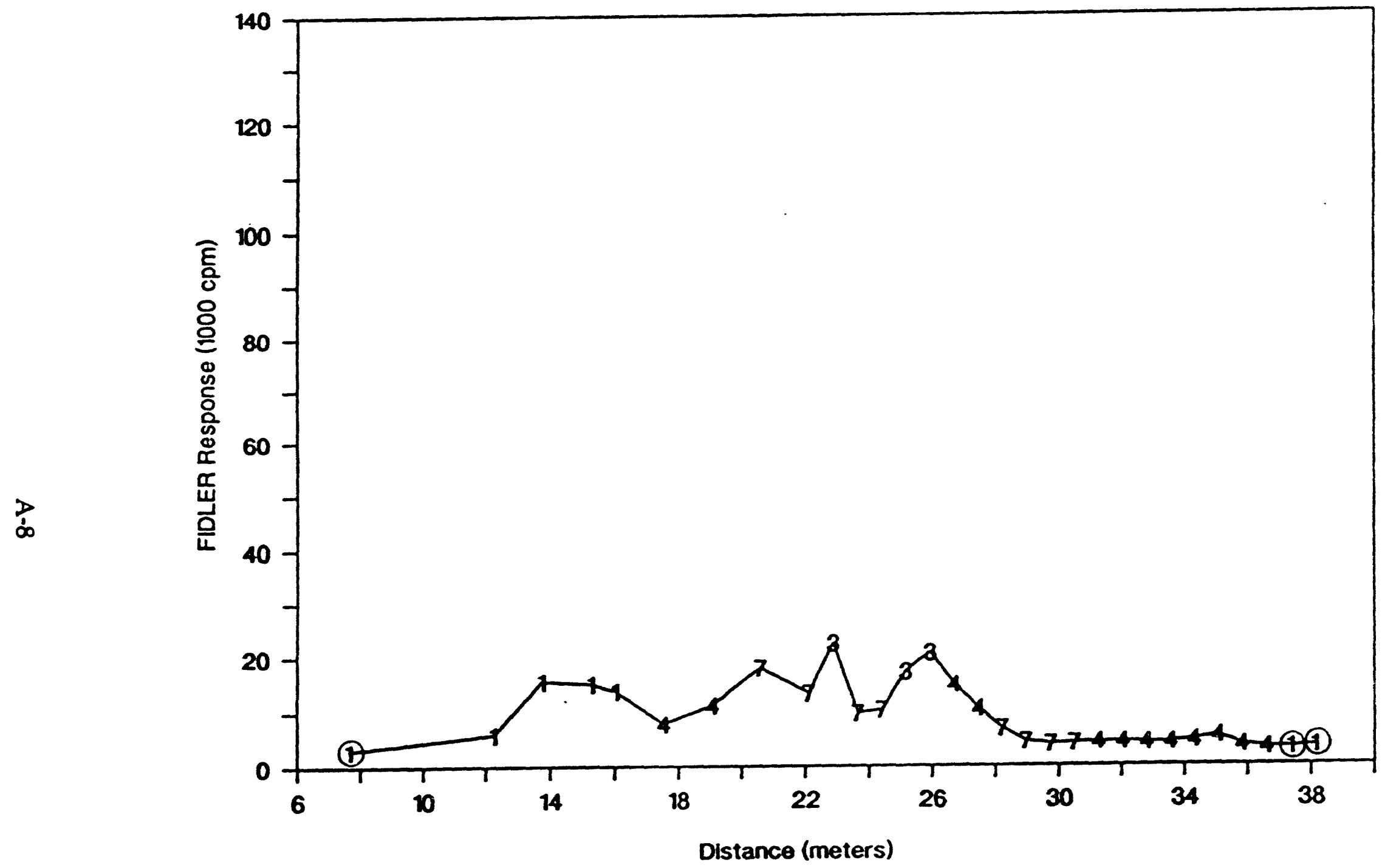

Figure A8. Area 11 Transect 36 - FIDLER measurements versus cross-stream position. The numbers printed at the plotted points are soil surface classification codes as listed in Table 2 in the text. The values for the circled numbers in the plot were used in estimating $q_{0}$. 


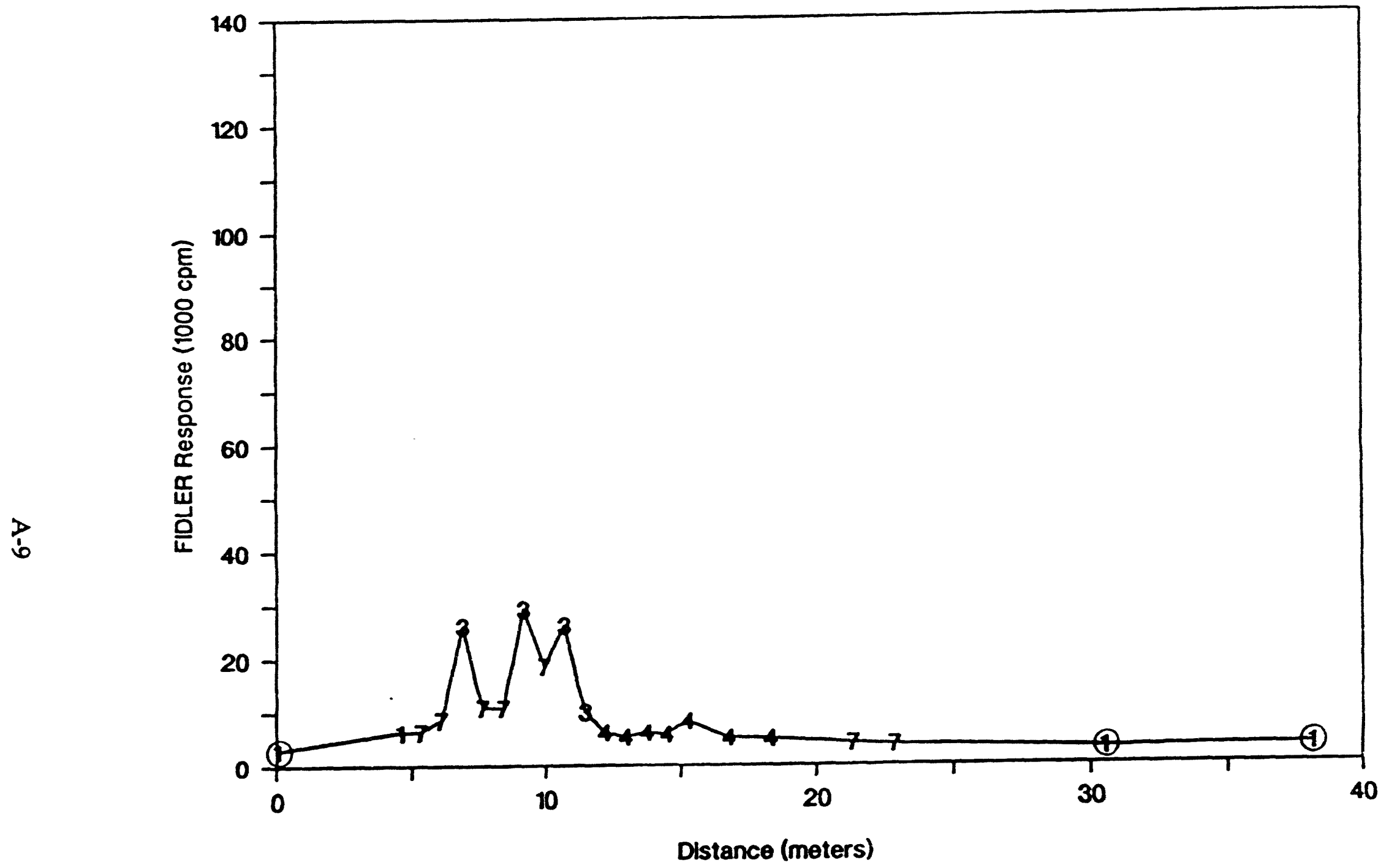

Figure A9. Area 11 Transect 38 - FIDLER measurements versus cross-stream position. The numbers printed at the plotted points are soil surface classification codes as listed in Table 2 in the text. The values for the circled numbers in the plot were used in estimating $q_{0}$. 


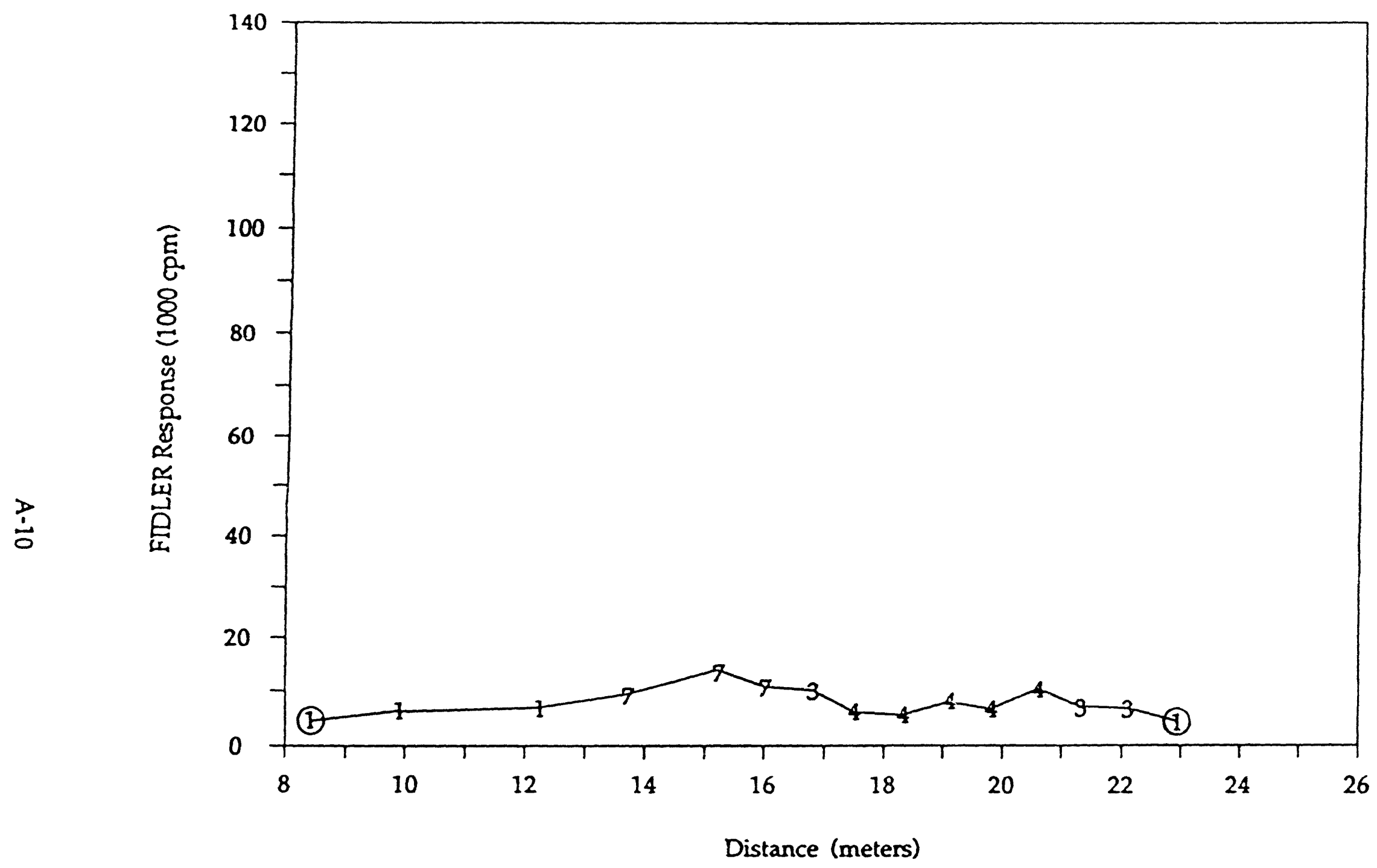

Figure A10. Area 11 Transect T - FIDLER measurements versus cross-stream position. The numbers printed at the plotted points are soil surface classification codes as listed in Table 2 in the text. The values for the circled numbers in the plot were used in estimating 90. 


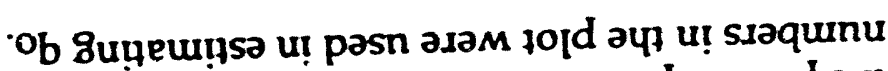

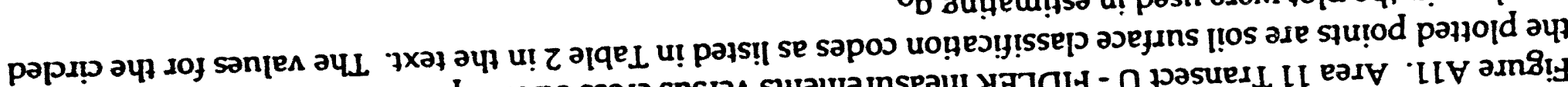

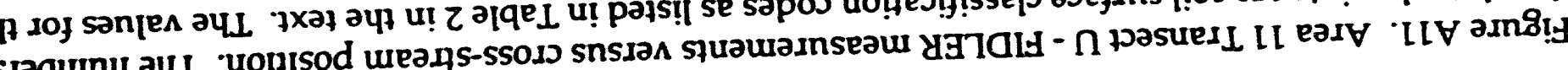

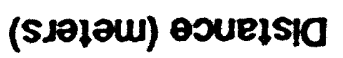

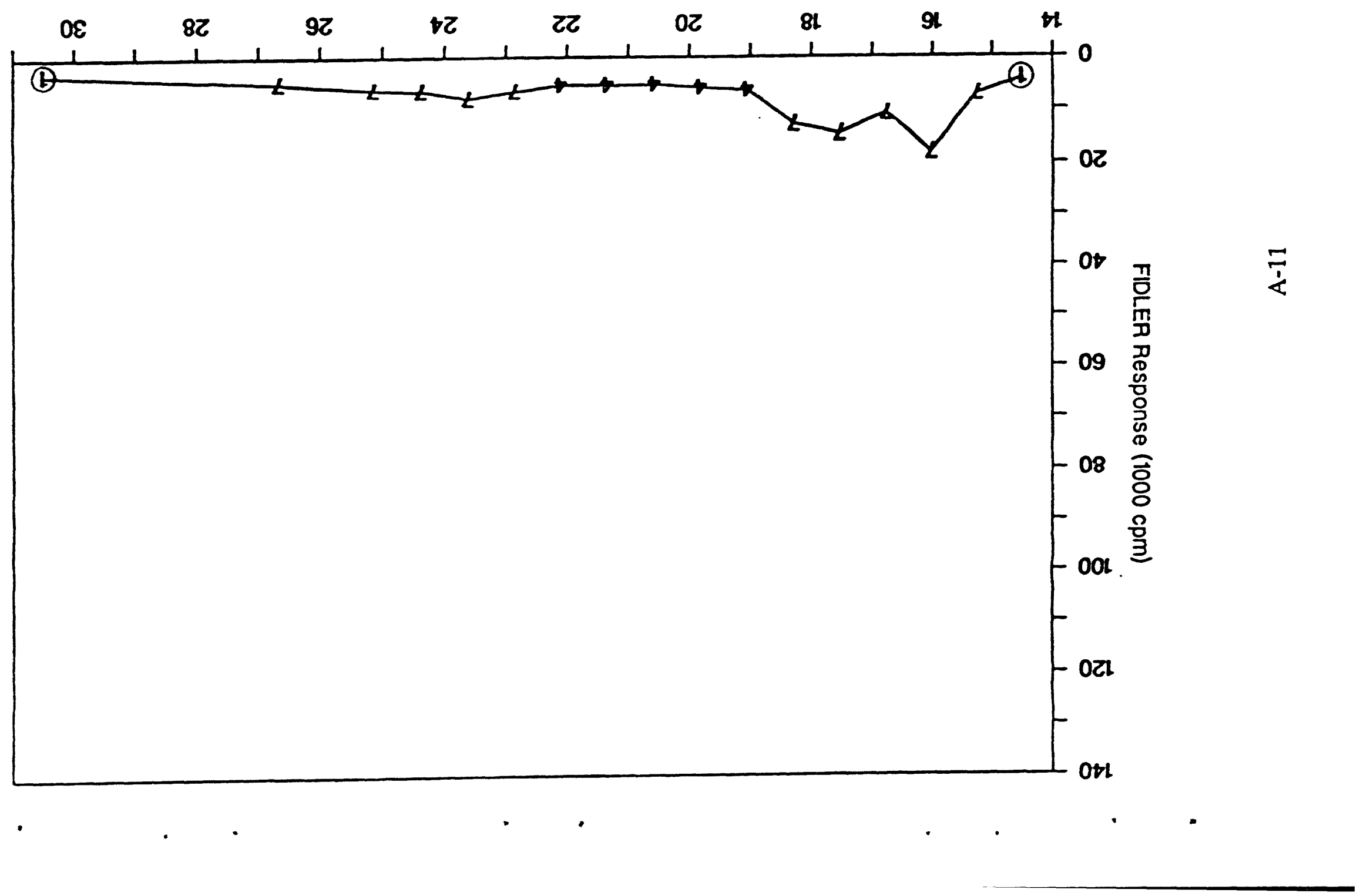




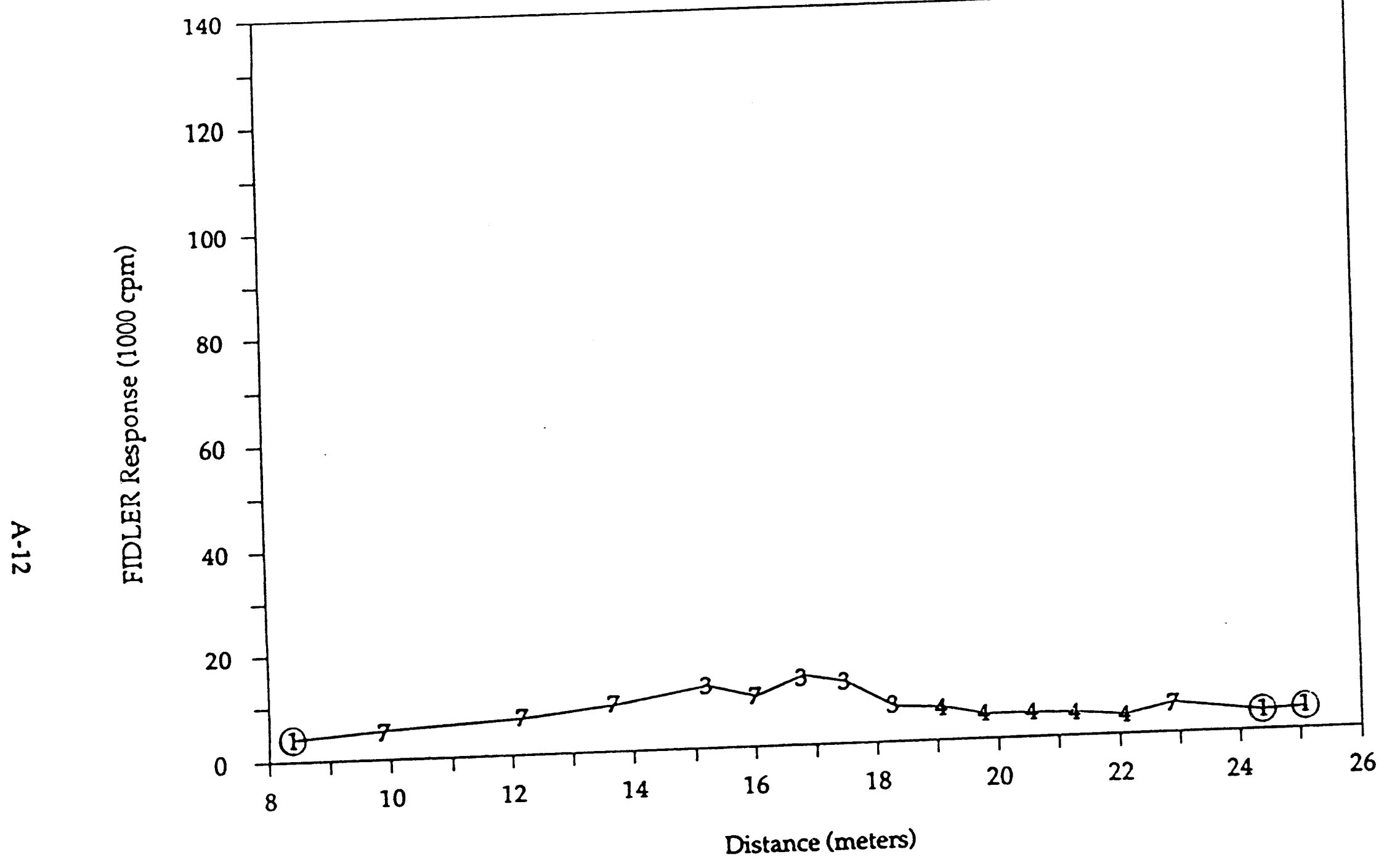

Area 11 Transect V - FIDLER measurements versus cross-stream position. The numbers printed at

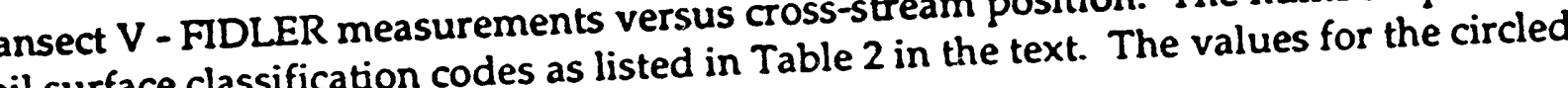
the plotted points are soil surface classification codes
numbers in the plot were used in estimating $q_{0}$. 


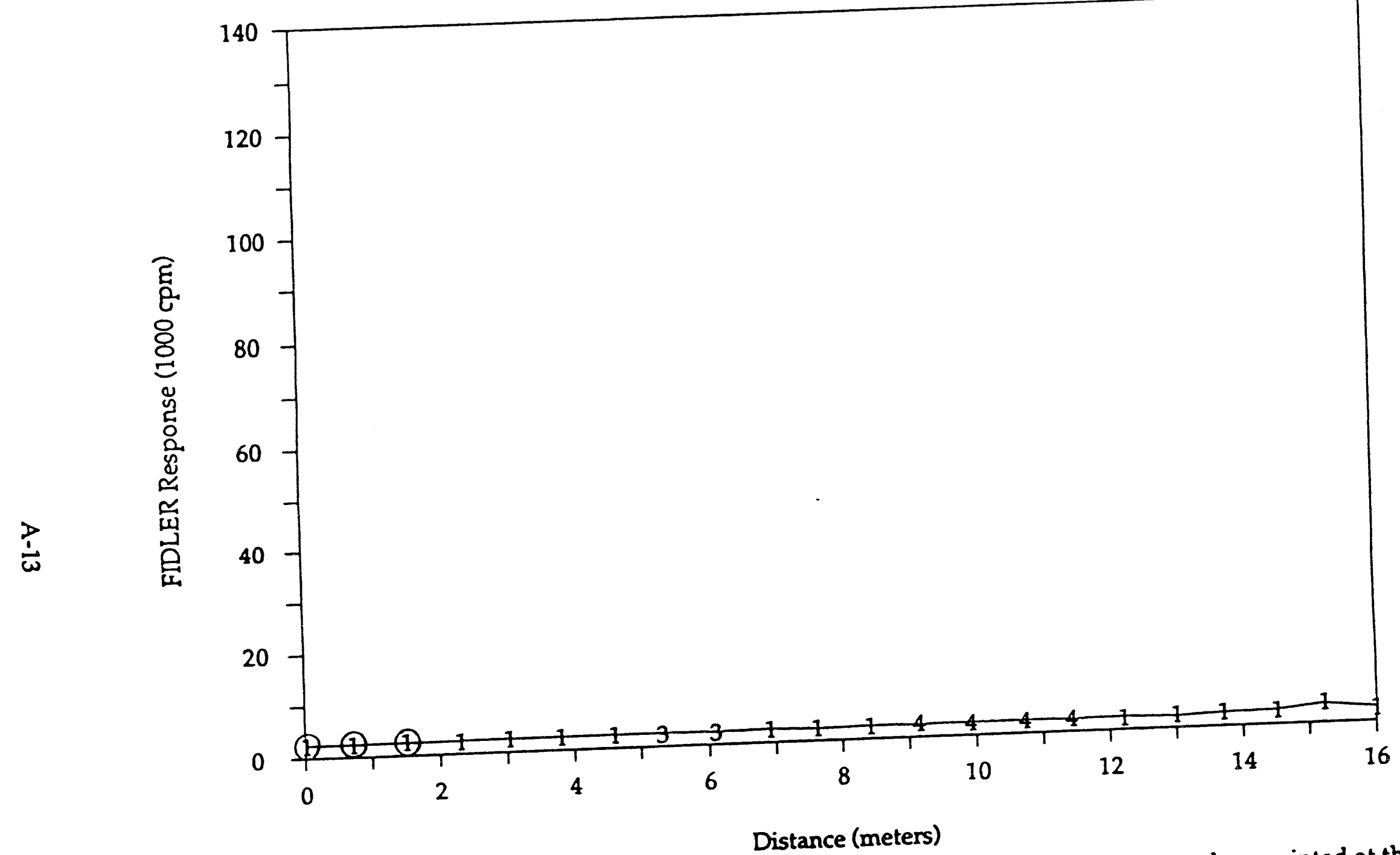
Figure A13. Area 11 Transect $Z$ - FIDLER measurements versus cross-stream position. The numbers printed numbers in the plot were used in estimating 90 . 
APPENDIX B: AREA 11 SAMPLING LOCATIONS AND

FIDLER MEASUREMENTS 
Appendix B: Area 11 Sampling Locations and FIDLER Measurements

\begin{tabular}{|c|c|c|c|c|}
\hline $\begin{array}{c}\text { Stake } \\
\text { Location }\end{array}$ & $\begin{array}{c}\text { Down- } \\
\text { stream (m) }\end{array}$ & $\begin{array}{c}\text { Cross- } \\
\text { Section (m) }\end{array}$ & $\begin{array}{c}\text { Surface } \\
\text { Description } 1\end{array}$ & $\begin{array}{l}\text { Counts } \\
\text { (cpm) }\end{array}$ \\
\hline $\begin{array}{r}29-\mathrm{E} \\
29-\mathrm{G} \\
29-\mathrm{I} \\
29-\mathrm{I}-7 \\
29-\mathrm{I}-8 \\
29-\mathrm{I}-9 \\
29-\mathrm{J} \\
29-\mathrm{J}-1 \\
29-\mathrm{J}-2 \\
29-\mathrm{J}-3 \\
29-\mathrm{J}-4 \\
29-\mathrm{J}-5 \\
29-\mathrm{J}-6 \\
29-\mathrm{J}-7 \\
29-\mathrm{J}-8 \\
29-\mathrm{J}-9 \\
29-\mathrm{K} \\
29-\mathrm{L} \\
29-\mathrm{M} \\
29-\mathrm{M}-1 \\
29-\mathrm{M}-2 \\
29-\mathrm{M}-3 \\
29-\mathrm{M}-4 \\
29-\mathrm{M}-5 \\
29-\mathrm{M}-6 \\
29-\mathrm{M}-7 \\
29-\mathrm{M}-8 \\
29-\mathrm{M}-9 \\
29-\mathrm{N} \\
29-\mathrm{N}-1 \\
29-\mathrm{N}-2 \\
29-\mathrm{N}-3 \\
29-\mathrm{N}-4 \\
29-\mathrm{N}-5 \\
29-\mathrm{N}-6 \\
29-\mathrm{N}-7 \\
29-\mathrm{N}-8 \\
29-\mathrm{N}-9 \\
29-0 \\
29-\mathrm{O}-1 \\
29-\mathrm{O}-2 \\
29-\mathrm{O}-3\end{array}$ & $\begin{array}{l}79 \\
79 \\
79 \\
79 \\
79 \\
79 \\
79 \\
79 \\
79 \\
79 \\
79 \\
79 \\
79 \\
79 \\
79 \\
79 \\
79 \\
79 \\
79 \\
79 \\
79 \\
79 \\
79 \\
79 \\
79 \\
79 \\
79 \\
79 \\
79 \\
79 \\
79 \\
79 \\
79 \\
79 \\
79 \\
79 \\
79 \\
79 \\
79 \\
79 \\
79 \\
79\end{array}$ & $\begin{array}{r}22.9 \\
38.1 \\
53.3 \\
58.7 \\
59.4 \\
60.2 \\
61.0 \\
61.7 \\
62.5 \\
63.2 \\
64.0 \\
64 \\
64.8 \\
65.5 \\
66.3 \\
67.1 \\
67.8 \\
67 \\
68.6 \\
76.2 \\
83.8 \\
84.6 \\
85\end{array}$ & $\begin{array}{l}1 \\
3 \\
1 \\
1 \\
1 \\
1 \\
1 \\
4 \\
4 \\
4 \\
4 \\
1 \\
1 \\
1 \\
3 \\
1 \\
1 \\
7 \\
4 \\
4 \\
4 \\
4 \\
4 \\
4 \\
4 \\
4 \\
4 \\
4 \\
4 \\
7 \\
7 \\
1 \\
1 \\
1 \\
1 \\
1 \\
1 \\
1 \\
1 \\
1 \\
1 \\
1\end{array}$ & $\begin{array}{r}7530{ }^{*} \\
6580{ }^{*} \\
15400 \\
48100 \\
55300 \\
37700 \\
83100 \\
91600 \\
99500 \\
102000 \\
119000 \\
40800 \\
19000 \\
18200 \\
19300 \\
23900 \\
24100 \\
20800 \\
13500 \\
13700 \\
8520 \\
8330 \\
7920 \\
4460 \\
6510 \\
9240 \\
13300 \\
19200 \\
23250 \\
23400 \\
19800 \\
19600 \\
23600 \\
26800 \\
27100 \\
31500 \\
20000 \\
35800 \\
29400 \\
27900 \\
24800 \\
25500\end{array}$ \\
\hline
\end{tabular}

1 Refers to soil surface classification code (see Table IV-2 in text).

* Values used to determine qo for transect. 


\begin{tabular}{|c|c|c|c|c|}
\hline $\begin{array}{l}\text { Stake } \\
\text { Location }\end{array}$ & $\begin{array}{c}\text { Down- } \\
\text { stream (m) S }\end{array}$ & $\begin{array}{c}\text { Cross- } \\
\text { Section (m) }\end{array}$ & $\begin{array}{c}\text { Surface } \\
\text { Description } 1\end{array}$ & $\begin{array}{l}\text { Counts } \\
\text { (cpm) }\end{array}$ \\
\hline $\begin{array}{r}30-M-8 \\
30-N\end{array}$ & $\begin{array}{l}88 \\
88\end{array}$ & $\begin{array}{l}89.9 \\
91.4\end{array}$ & $\begin{array}{l}7 \\
7\end{array}$ & $\begin{array}{l}19800 \\
21000\end{array}$ \\
\hline $\begin{array}{r}31-E \\
31-F \\
31-G \\
31-G-1 \\
31-G-2 \\
31-G-3 \\
31-G-4 \\
31-G-5 \\
31-G-6 \\
31-G-7 \\
31-G-8 \\
31-G-9 \\
31-H \\
31-H-1 \\
31-H-2 \\
31-H-3 \\
31-H-4 \\
31-H-5 \\
31-H-6 \\
31-H-7 \\
31-H-8 \\
31-H-9 \\
31-I \\
31-I-1 \\
31-I-2 \\
31-I-3 \\
31-I-4 \\
31-I-5 \\
31-I-6 \\
31-I-7 \\
31-I-8 \\
31-I-9 \\
31-J \\
31-J-1 \\
31-J-2 \\
31-J-3 \\
31-J-4 \\
31-J-5 \\
31-J-6 \\
31-J-7 \\
31-J-8\end{array}$ & $\begin{array}{l}97 \\
97 \\
97 \\
97 \\
97 \\
97 \\
97 \\
97 \\
97 \\
97 \\
97 \\
97 \\
97 \\
97 \\
97 \\
97 \\
97 \\
97 \\
97 \\
97 \\
97 \\
97 \\
97 \\
97 \\
97 \\
97 \\
97 \\
97 \\
97 \\
97 \\
97 \\
97 \\
97 \\
97 \\
97 \\
97 \\
97 \\
97 \\
97 \\
97 \\
97\end{array}$ & $\begin{array}{l}22.9 \\
30.5 \\
38.1 \\
38.9 \\
39.6 \\
40.4 \\
41.1 \\
41.9 \\
42.7 \\
43.4 \\
44.2 \\
45.0 \\
45.7 \\
46.5 \\
47.2 \\
48.0 \\
48.8 \\
49.5 \\
50.3 \\
51.1 \\
51.8 \\
52.6 \\
53.3 \\
54.1 \\
54.9 \\
55.6 \\
56.4 \\
57.2 \\
57.9 \\
58.7 \\
59.4 \\
60.2 \\
61.0 \\
61.7 \\
62.5 \\
63.2 \\
64.0 \\
64.8 \\
65 \\
67 \\
6\end{array}$ & $\begin{array}{l}1 \\
1 \\
3 \\
1 \\
3 \\
7 \\
1 \\
1 \\
1 \\
1 \\
1 \\
1 \\
1 \\
7 \\
3 \\
3 \\
4 \\
4 \\
4 \\
3 \\
7 \\
7 \\
3 \\
4 \\
4 \\
7 \\
7 \\
7 \\
7 \\
7 \\
7 \\
7 \\
7 \\
1 \\
1 \\
3 \\
7 \\
7 \\
4 \\
4 \\
4\end{array}$ & $\begin{array}{r}3820{ }^{\star} \\
5280 \star \\
28800 \\
22800 \\
31900 \\
21500 \\
27400 \\
29400 \\
28400 \\
23300 \\
38600 \\
56500 \\
40600 \\
70000 \\
128000 \\
137000 \\
75000 \\
81600 \\
84100 \\
47600 \\
33600 \\
61300 \\
127000 \\
99000 \\
52300 \\
11400 \\
5810 \\
7580 \\
7060 \\
8020 \\
8110 \\
8670 \\
7730 \\
5820 \\
9270 \\
19800 \\
5710 \\
4310 \\
6470 \\
7110 \\
6290\end{array}$ \\
\hline
\end{tabular}

1 Refers to soil surface classification code (see Table IV-2 in text).

* Values used to determine qo for transect. 


\begin{tabular}{|c|c|c|c|c|}
\hline $\begin{array}{l}\text { Stake } \\
\text { Location }\end{array}$ & $\begin{array}{c}\text { Down- } \\
\text { stream (m) }\end{array}$ & $\begin{array}{c}\text { Cross- } \\
\text { Section (m) }\end{array}$ & $\begin{array}{c}\text { Surface } \\
\text { Description } 1\end{array}$ & $\begin{array}{l}\text { Counts } \\
\text { (cpm) }\end{array}$ \\
\hline $\begin{array}{r}32-I-4 \\
32-I-5 \\
32-I-6 \\
32-I-7 \\
32-I-8 \\
32-I-9 \\
32-J \\
32-J-1 \\
32-J-2 \\
32-J-3 \\
32-J-4\end{array}$ & $\begin{array}{l}106 \\
106 \\
106 \\
106 \\
106 \\
106 \\
106 \\
106 \\
106 \\
106 \\
106\end{array}$ & $\begin{array}{l}56.4 \\
57.1 \\
57.9 \\
58.7 \\
59.4 \\
60.2 \\
61.0 \\
61.7 \\
62.5 \\
63.2 \\
6 . .0\end{array}$ & $\begin{array}{l}4 \\
4 \\
4 \\
4 \\
4 \\
4 \\
7 \\
7 \\
1 \\
1 \\
1\end{array}$ & $\begin{array}{l}5100 \\
6790 \\
4580 \\
4750 \\
5190 \\
6010 \\
9100 \\
7480 \\
5660^{\star} \\
4800^{\star} \\
3720^{\star}\end{array}$ \\
\hline $\begin{array}{r}33-E \\
33-E-9 \\
33-F \\
33-F-1 \\
33-F-2 \\
33-F-3 \\
33-F-4 \\
33-F-5 \\
33-F-6 \\
33-F-7 \\
33-F-8 \\
33-F-9 \\
33-G \\
33-H \\
33-H-1 \\
33-H-2 \\
33-H-3 \\
33-H-4 \\
33-H-5 \\
33-H-6 \\
33-H-7 \\
33-H-8 \\
33-H-9 \\
33-I \\
33-I-1 \\
33-I-2 \\
33-I-3 \\
33-I-4 \\
33-J \\
33-K \\
33-M\end{array}$ & $\begin{array}{l}115 \\
115 \\
115 \\
115 \\
115 \\
115 \\
115 \\
115 \\
115 \\
115 \\
115 \\
115 \\
115 \\
115 \\
115 \\
115 \\
115 \\
115 \\
115 \\
115 \\
115 \\
115 \\
115 \\
115 \\
115 \\
115 \\
115 \\
115 \\
115 \\
115 \\
115\end{array}$ & $\begin{array}{l}22.9 \\
29.7 \\
30.5 \\
31.2 \\
32.0 \\
32.8 \\
33.5 \\
34.3 \\
35.1 \\
35.8 \\
36.6 \\
37.3 \\
38.1 \\
45.7 \\
46.5 \\
47.2 \\
48.0 \\
48.8 \\
49.5 \\
50.3 \\
51.1 \\
51.8 \\
52.6 \\
53.3 \\
54.1 \\
54.9 \\
55.6 \\
56.4 \\
61.0 \\
68.6 \\
83.8\end{array}$ & $\begin{array}{l}1 \\
1 \\
3 \\
7 \\
7 \\
4 \\
4 \\
4 \\
3 \\
7 \\
7 \\
3 \\
7 \\
7 \\
7 \\
7 \\
7 \\
7 \\
4 \\
4 \\
4 \\
4 \\
4 \\
4 \\
4 \\
7 \\
7 \\
1 \\
3 \\
3 \\
1\end{array}$ & $\begin{array}{c}5495^{\star} \\
28100 \\
51500 \\
40800 \\
35900 \\
34000 \\
25300 \\
19200 \\
20500 \\
8300 \\
5580 \\
8810 \\
11500 \\
5390 \\
5390 \\
5940 \\
4660 \\
5620 \\
4870 \\
5350 \\
6350 \\
5830 \\
4830 \\
4805 \\
5610 \\
5490 \\
4170 \\
5540 \star \\
5280^{\star} \\
6260 \star \\
7610^{\star}\end{array}$ \\
\hline
\end{tabular}

1 Refers to soil surface classification code (see Table IV-2 in text).

* Values used to determine qo for transect. 


\begin{tabular}{|c|c|c|c|c|}
\hline $\begin{array}{l}\text { Stake } \\
\text { Location }\end{array}$ & $\begin{array}{c}\text { Down- } \\
\text { stream (m) }\end{array}$ & $\begin{array}{c}\text { Cross- } \\
\text { Section (m) }\end{array}$ & $\begin{array}{c}\text { Surface } \\
\text { Description } 1\end{array}$ & $\begin{array}{l}\text { Counts } \\
\text { (cpm) }\end{array}$ \\
\hline $\begin{array}{r}34-H-9 \\
34-I\end{array}$ & $\begin{array}{l}124 \\
124\end{array}$ & $\begin{array}{l}52.6 \\
53.3\end{array}$ & $\begin{array}{l}1 \\
1\end{array}$ & $\begin{array}{l}4290^{*} \\
4510^{*}\end{array}$ \\
\hline $\begin{array}{r}35-E \\
35-E-1 \\
35-E-3 \\
35-E-5 \\
35-E-7 \\
35-E-8 \\
35-E-9 \\
35-F \\
35-F-1 \\
35-F-2 \\
35-F-3 \\
35-F-4 \\
35-F-5 \\
35-F-6 \\
35-F-7 \\
35-F-8 \\
35-F-9 \\
35-G \\
35-G-1 \\
35-G-2 \\
35-G-3 \\
35-G-4 \\
35-G-5 \\
35-G-6 \\
35-G-7 \\
35-G-8 \\
35-G-9 \\
35-H \\
35-H-1 \\
35-H-2 \\
35-K \\
35-M\end{array}$ & $\begin{array}{l}133 \\
133 \\
133 \\
133 \\
133 \\
133 \\
133 \\
133 \\
133 \\
133 \\
133 \\
133 \\
133 \\
133 \\
133 \\
133 \\
133 \\
133 \\
133 \\
133 \\
133 \\
133 \\
133 \\
133 \\
133 \\
133 \\
133 \\
133 \\
133 \\
133 \\
133 \\
133\end{array}$ & $\begin{array}{l}22.9 \\
23.6 \\
25.1 \\
26.7 \\
28.2 \\
29.0 \\
29.7 \\
30.5 \\
31.2 \\
32.0 \\
32.8 \\
33.5 \\
34.3 \\
35.1 \\
35.8 \\
36.6 \\
37.3 \\
38.1 \\
38.9 \\
39.6 \\
40.4 \\
41.1 \\
41.9 \\
42.7 \\
43.4 \\
44.2 \\
45.0 \\
45.7 \\
46.5 \\
47.2 \\
68.6 \\
83.8\end{array}$ & $\begin{array}{l}3 \\
7 \\
7 \\
7 \\
7 \\
7 \\
7 \\
7 \\
7 \\
4 \\
4 \\
7 \\
7 \\
7 \\
7 \\
7 \\
7 \\
3 \\
7 \\
4 \\
4 \\
4 \\
4 \\
4 \\
4 \\
7 \\
7 \\
1 \\
1 \\
1 \\
1 \\
1\end{array}$ & $\begin{array}{l}17400 \\
11300 \\
15100 \\
16800 \\
17100 \\
13800 \\
16300 \\
20600 \\
14500 \\
12000 \\
17700 \\
12200 \\
5980 \\
7220 \\
6160 \\
5680 \\
5540 \\
6300 \\
4710 \\
5210 \\
4130 \\
4310 \\
5230 \\
5730 \\
5640 \\
4950 \\
3960 \\
4680 \star \\
4790 \star \\
3860 \star \\
4630 \star \\
4090 \star\end{array}$ \\
\hline $\begin{array}{r}36-C \\
36-C-6 \\
36-C-8 \\
36-D \\
36-D-1 \\
36-D-3 \\
36-D-5 \\
36-D-7\end{array}$ & $\begin{array}{l}141 \\
141 \\
141 \\
141 \\
141 \\
141 \\
141 \\
141\end{array}$ & $\begin{array}{r}7.6 \\
12.2 \\
13.7 \\
15.2 \\
16.0 \\
17.5 \\
19.1 \\
20.6\end{array}$ & $\begin{array}{l}1 \\
1 \\
1 \\
1 \\
1 \\
4 \\
4 \\
7\end{array}$ & $\begin{array}{r}3010 * \\
5930 \\
15500 \\
15000 \\
13800 \\
7900 \\
11200 \\
17900\end{array}$ \\
\hline
\end{tabular}

1 Refers to soil surface classification code (see Table IV-2 in text).

* Values used to determine qo for transect. 


\begin{tabular}{|c|c|c|c|c|}
\hline $\begin{array}{l}\text { Stake } \\
\text { Location }\end{array}$ & $\begin{array}{c}\text { Down- } \\
\text { stream (m) }\end{array}$ & $\begin{array}{c}\text { Cross- } \\
\text { Section (m) }\end{array}$ & $\begin{array}{c}\text { Surface } \\
\text { Description } 1\end{array}$ & $\begin{array}{l}\text { Counts } \\
\text { (cpm) }\end{array}$ \\
\hline $\begin{array}{l}38-F \\
38-G\end{array}$ & $\begin{array}{l}159 \\
159\end{array}$ & $\begin{array}{l}30.5 \\
38.1\end{array}$ & $\begin{array}{l}1 \\
1\end{array}$ & $\begin{array}{l}2990^{\star} \\
3350^{\star}\end{array}$ \\
\hline $\begin{array}{c}T-99 \\
T-0 \\
T-1 \\
T-2 \\
T-3 \\
T-4 \\
T-5 \\
T-6 \\
T-7 \\
T-8 \\
T-9 \\
T-10 \\
T-11 \\
T-12 \\
T-14\end{array}$ & $\begin{array}{l}175 \\
175 \\
175 \\
175 \\
175 \\
175 \\
175 \\
175 \\
175 \\
175 \\
175 \\
175 \\
175 \\
175 \\
175\end{array}$ & $\begin{array}{l}14.5 \\
15.2 \\
16.0 \\
16.8 \\
17.5 \\
18.3 \\
19.1 \\
19.8 \\
20.6 \\
21.3 \\
22.1 \\
22.9 \\
23.6 \\
24.4 \\
25.9\end{array}$ & $\begin{array}{l}1 \\
1 \\
1 \\
7 \\
7 \\
7 \\
3 \\
4 \\
4 \\
4 \\
4 \\
4 \\
3 \\
3 \\
1\end{array}$ & $\begin{array}{c}4750 \star \\
6110 \\
6950 \\
9420 \\
14000 \\
10900 \\
10200 \\
6100 \\
5730 \\
8090 \\
6660 \\
10500 \\
7270 \\
6880 \\
4240 *\end{array}$ \\
\hline $\begin{array}{c}U-99 \\
U-0 \\
U-1 \\
U-2 \\
U-3 \\
U-4 \\
U-5 \\
U-6 \\
U-7 \\
U-8 \\
U-9 \\
U-10 \\
U-11 \\
U-12 \\
U-13 \\
U-15 \\
U-20\end{array}$ & $\begin{array}{l}191 \\
191 \\
191 \\
191 \\
191 \\
191 \\
191 \\
191 \\
191 \\
191 \\
191 \\
191 \\
191 \\
191 \\
191 \\
191 \\
191\end{array}$ & $\begin{array}{l}14.5 \\
15.2 \\
16.0 \\
16.8 \\
17.5 \\
18.3 \\
19.1 \\
19.8 \\
20.6 \\
21.3 \\
22.1 \\
22.9 \\
23.6 \\
24.4 \\
25.1 \\
26.7 \\
30.5\end{array}$ & $\begin{array}{l}1 \\
7 \\
7 \\
7 \\
7 \\
7 \\
4 \\
4 \\
4 \\
4 \\
4 \\
7 \\
7 \\
7 \\
7 \\
7 \\
1\end{array}$ & $\begin{array}{r}4050 \star \\
7110 \\
18100 \\
10900 \\
14600 \\
12900 \\
6180 \\
5720 \\
5090 \\
5280 \\
5300 \\
6460 \\
7940 \\
6400 \\
6150 \\
4890 \\
3030 \star\end{array}$ \\
\hline $\begin{array}{l}V-91 \\
V-93 \\
V-96 \\
V-98 \\
V-0 \\
V-1 \\
V-2\end{array}$ & $\begin{array}{l}264 \\
264 \\
264 \\
264 \\
264 \\
264 \\
264\end{array}$ & $\begin{array}{r}8.4 \\
9.9 \\
12.2 \\
13.7 \\
15.2 \\
16.0 \\
16.8\end{array}$ & $\begin{array}{l}1 \\
7 \\
7 \\
7 \\
3 \\
7 \\
3\end{array}$ & $\begin{array}{r}3830 \\
5360 \\
6850 \\
8710 \\
11800 \\
9440 \\
13100\end{array}$ \\
\hline
\end{tabular}

1 Refers to soil surface classification code (see Table IV-2 in text).

* Values used to determine go for transect. 
APPENDIX C: DETAILED PROCEDURES FOR THE FIDLER 
BECAMP-DP-401, RO

BECAMP Quality Assurance Detailed Procedure for FIDLER Set-up and Calibration

\author{
Prepared by \\ Scott E. Patton \\ Environmental Sciences Division \\ Lawrence Livermore National Laboratory
}

October 1990

Prepared for the U.S. Department of Energy, Nevada Operations Office, sponsored Basic Environmental Compliance and Monitoring Program 
BECAMP DETAILED PROCEDURE APPROVAL

Effective Date April 27, 1992

TASK NO_ 1 DP IDENTIFIER_BECAMP-DP-401

PRINCIPAL INVESTIGATOR_Joseph H. Shinn

ORGANIZATION_Lawrence Livermore National Laboratory

TITLE: BECAMP Quality Assurance Detailed Procedure for FIDLER Set-up and Calibration

SIGNATURES:

QCP civeren.

Preparer: S.E. Patton

Toinh it fhaion

f. ConcalRevewer: J.H. Shinn

L. is

Quality Assurance E. H. Essington

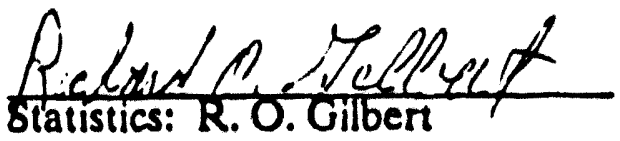

COPIES TO:

J. B. Hall, Chief, Technology Development Branch US Department of Energy, NVO

L. R. Anspaugh, BECAMP Scientific Director

Lawrence Livermore National Laboratory
Date isw?

?5/Ei-92

Date

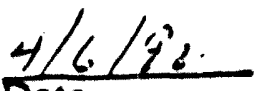

Date

Qenei 10,1993

April 27, 1992

Date Sent

Apri1 27, 1992

Date Sent 


\section{PURPOSE}

The following procedure is to be used in the set-up and calibration of the FIDLER (field instrument for the detection of low-energy radiation) detector system for in situ measurements of americium-241 (Am-241) activity in soil. This procedure (DP-401) is the first of three (BECAMP) Basic Environmental Compliance and Monitoring Program Quality Assurance Detailed Procedures (DPs) for quantifying radionuclide concentrations in soils from in situ measurements with a FIDLER. The second DP (DP402 ) is the procedure for taking in situ measurements with the FIDLER and the third $D P(D P-403)$ is the procedure for quantifying radionuclide concentrations from in situ FIDLER measurements of radionuclide activity in soil. This FIDLER calibration procedure is divided into two parts: the pulse calibration of the analyzer and FIDLER probe for specific energy responses and the determination of the angular response and counting efficiency of the FIDLER to a specific radionuclide source.

This detailed procedure fulfills a requirement of the BECAMP Quality Assurance Plan (Essington 1989). Detailed procedures define technical requirements, constraints, and procedural steps of scientific activities that are to be conducted by BECAMP Investigators. The DP must be reviewed for appropriate quality-assurance and statistical considerations and must be approved by BECAMP authorities as designated in the Quality Assurance Plan.

\section{SCOPE}

The set-up and calibration procedures described in this BECAMP Quality Assurance DP are to be used for calibrating a FIDLER detector system (a FIDLER scintillation detector and pulse-rate analyzer) for BECAMP in situ measurements for the determination of Am-241 activity in soils of the NTS and its environs. (In this document and unless otherwise stated, FIDLER will refer to the FIDLER detector system comprised of a FIDLER scintillation detector and accompanying pulse-rate analyzer). There are two parts in the FIDLER calibration DP; Procedure 1, Calibration of the Pulse-Rate Analyzer, sets the analyzer and FIDLER detector for the detection of $60 \mathrm{keV}$ gamma rays emitted from Am-241, and Procedure 2, Determination of the FIDLER Counting Efficiency and Angular Response Measurements, determines the angular response and counting efficiency of a FIDLER to a specific radionuclide source. These measurements are used in a mathematical computer code (described in DP-403 and Couveia 1991) for quantifying soil radionuclide activity on the basis of detector geometries and radionuclide source distributions in the soil. The field monitoring protocol provided by the BECAMP Principal Investigator will add specifics not covered in this DP or variations to this DP.

This DP is specific for the set-up and calibration of the Eberline ESP-2 Smart Portable radiation survey instrument in conjunction with the Bicron FIDLER scintillation detector. The ESP-2 analyzer has many options and can be relatively 
complex to operate. However, due to its computerized nature it can also be extremely easy to use in the automatic inquiry mode. It is not the intent of this DP to provide an operating manual on the ESP-2 instrument. The use and operation of the ESP-2 can be found in "Model ESP-2 Eberline Smart Portable Technical Manual," December 1986, from Eberline. The ESP-2 operating manual can be found in the ESP-2/FIDLER carrying case.

\section{DEFINITIONS}

Analyzer - a pulse-rate meter that converts signals from a radiation-detection probe; specifically an Eberline ESP-2 Smart Portable Survey instrument with a PHA board.

Calibration Rig - A stainless steel bar in a half-circle shape, radius of one-meter, rotated by a motor at approximately 28 revolutions-per-minute that is used to determine the counting efficiency and angular response of a FIDLER. A calibration source is attached at different angles along the bar so that upon rotation of the bar the source scribes a circle about the axis of rotation (which is also the central axis of the detector). The rig can be obtained from Lawrence Livermore National Laboratory (LLNL), Environmental Sciences Division.

Calibration Stand - A stand for the FIDLER detector which centers the detector above the calibration rig on the axis of rotation, one-meter from the base and arm of the rig. The calibration stand can be a one-time fabrication by the person calibrating the FIDLER or it can be obtained from LLNL, Environmental Sciences Division.

Check source - A known amount of a specific radionuclide mounted on a small disc. The source should be calibrated for the quantity of material (usually in $\mu \mathrm{Ci}$ ) on the disc; a NIST-traceable source would be preferred. The date of calibration should also be known. For the purposes of this DP, the following sources and source strength are required:

$$
\begin{aligned}
& \text { Americium-241, } \sim 10 \mu \mathrm{Ci} \text {, and } \\
& \text { Americium-241, } \sim 100 \mu \mathrm{Ci} \text {. }
\end{aligned}
$$

FIDLER Detector - a scintillation probe for the detection of low-energy radiation, specifically a Bicron probe, Model G5 "FIDLER" containing a 5 -inch by 0.063 -inch Nal crystal.

FIDLER Stand - A metal tripod stand that supports the FIDLER $30 \mathrm{~cm}$ above the soil surface. The FIDLER stand can be obtained from Reynolds Electric \& Engineering Company (REECo) Soils Laboratory, at the Nevada Test Site. 


\section{RESPONSIBILITIES AND TRAINING}

In the current structure of the BECAMP program at the NTS (Patton 1990), the following persons/offices have the responsibility for calibrating the FIDLER detector and ESP-2 analyzer. REECo's Instrumentation Laboratory at the NTS is responsible for setting the analyzer high voltage and determine the operating voltage of the FIDLER (Procedure 1). REECo's Soils Laboratory will be responsible for the setup of the analyzer and the FIDLER counting-efficiency check and for determination of the FIDLER angular response (Procedure 2).

The calibration procedure is to be conducted by persons familiar with a FIDLER detector and ESP-2 analyzer. Those persons involved with the calibration and set-up of the FIDLER will have working knowledge of the calibration procedures prior to actual calibration of a FIDLER and analyzer. The responsibility of training belongs to the Principal Investigator of BECAMP Task 1 (currently Joseph H. Shinn of Lawrence Livermore National Laboratory) and can be delegated to others trained by the Principal Investigator.

\section{RECORD KEEPING}

Each step in this calibration procedure will be signed off by the person performing the procedure. The Calibration Data and Sign-Off form (Attachment 1) will accompany the FIDLER through the calibration procedures. The completed data and sign-off form will accompany the field data to the Principal Investigator.

\section{PROCEDURE 1 - Calibration of Pulse-Rate Analyzer}

This procedure includes the setting of the ESP-2 pulse-rate analyzer high voltage, setting of the gain and threshold, and determination of a $50 \%$ window for threshold voltage, analyzer setup, and determining the FIDLER operating voltage. An Am-241 check source is used to set the operating voltage of the FIDLER.

The high voltage should be checked at least once a year by REECo's Instrumentation Laboratory. They should also check the operating voltage, gain and threshold, and determination of a $50 \%$ window for threshold voltage on a regularly scheduled basis (as determined by REECo's standard operating procedures) or prior to the use of the FIDLER in BECAMP investigations.

Source: Am-241, $\sim 10 \mu \mathrm{Ci}$ check source.

Definitions:

Access Cover - This cover provides access to the internal calibration controls of the ESP-2 and is found on the right side of the instrument. 
Dip Switches - There are six dip switches located next to the potentiometers under the access cover. The purpose of four switches is to adjust the speaker repetition rate. They divide the speaker rate by the factors indicated. The last two dip switches are for automatic testing of the instrument at the factory or designated Eberline repair facility. When any switch is down, the switch is in the circuit.

Potentiometers - located inside the access cover, all potentiometers are labeled and are discussed in detail in the ESP-2 Technical Manual. The window potentiometer is located on the PHA board and is accessed through the hole in the bottom cover of the instrument.

\section{A. High Voltage Calibration}

The ESP-2 high voltage is set before the analyzer is shipped from the manufacturer. One may check the high-voltage setting by connecting a highimpedance voltmeter (positive lead) to the central pin and the black ground lead to the outside of the MHV connector on the front of the analyzer. If the voltage reading on the voltmeter is different ( \pm 100 volts) than the voltage displayed on the ESP-2, then the voltage should be changed and viewed on the ESP-2 and measured on the voltmeter. Note that when adjusting or changing the high voltage, it is very difficult to set at exact voltage increments, so set the high voltage as close to the desired value as possible.

The method for changing the high voltage is as follows:

1. Turn the instrument on and then depress the "Mode" and "Reset" buttons simultaneously. The display then shows four different options: "R/LOG", "-/PARAMS", "+/OPER", "S/CLOCK". To select an option, press the button of the letter or symbol preceding the function. Therefore, to get to the "Parameters" section, depress the "-" button and the display will show the most recent detector used with ESP-2. Push the "+" button to accept the other items until the high voltage ("HV") is indicated. By simultaneously depressing the "Reset" and the " + " or "-" button the high voltage may be increased or decreased, respectively. The value is displayed on the liquid crystal display (LCD) of the analyzer and the measured value is displayed on the voltmeter.

2. Change the voltage to 600 volts and take a voltage measurement and record the results. Repeat the above procedure in 100 volt increments from 600 to 1400 volts. The measured data should agree with displayed values within \pm 50 volts. If the results do not agree, adjust the calibration as per paragraph (3) below.

3. If the voltage calibration exceeds the above limit then adjust the voltage as follows: adjust the high voltage display on the analyzer to 600 volts by 
depressing the "Reset" and " + " or "-" buttons simultaneously. Adjust the HV LEVEL (R68 potentiometer inside side access) until the measured voltage is 600 and then adjust the high voltage read on the LCD display to 1800 volts by depressing the "Reset" and "+" buttons. Next adjust the HV GAIN (R66 potentiometer inside side access) until the measured voltage is 1800 volts. Then change the voltage displayed on the ESP-2 by depressing the "Reset" and "-" buttons simultaneously until 600 volts are displayed. Then readjust HV LEVEL until the measured high voltage is 600 volts. Then readjust the high voltage display on the ESP-2 by depressing the "Reset" and "+" simultaneously until 1800 volts are displayed and readjust the HV GAIN until the output measures 1800 volts. Repeat this procedure until the measured values agree with the displayed values.

\section{B. Dead Time and Window Adjustment for the Bicron FIDLER Probe}

The overall instrument gain and discriminator setting should be adjusted for the FIDLER before an operating voltage is selected. It should be noted that section V.A.3. of the ESP-2 technical manual is not applicable to analyzers with a pulse-height analyzer (PHA) board. In addition, for instruments utilizing the PHA mode, the deadtime (DT) selection is essentially nonfunctional.

1. Dead time correction factor adjustment. The purpose of this adjustment is to correct for the number of gross counts arriving at the detector simultaneously at high count rates. If, in the gross count rate mode (GROSS on the PHA-GROSS switch located on the face of the ESP-2 analyzer), the number of counts is high and dead time is not correctly set, then the number of counts displayed may not be correct. Set the dead time (DT) to 1.4-0.5 $\left(1.4 \times 10^{-5}\right) \mathrm{sec}$. This is accomplished by turning on the instrument and simultaneously pushing the "RESET" and "MODE" buttons. Select the "-" button to select the "Parameters" and then push the "+" button to accept the values until "DT" is displayed on the LCD. At this point adjust the dead time by simultaneously pushing the "RESET" and "+" or "-" buttons to either increase or decrease the value to $1.4-05\left(1.4 \times 10^{-5}\right) \mathrm{sec}$.

2. Window Adjustment. This method uses a voltage pulser and adjusts the ESP-2 for a voltage range of 0 to $1 \mathrm{MeV}$ with a $50 \%$ window. The definition of a window (in percent) is

$$
\% \text { window }=100 *(\mathrm{Vu}-\mathrm{Vl}) / \mathrm{Vl} \text {, }
$$

where $\mathrm{Vu}=$ upper threshold voltage and $\mathrm{Vl}=$ lower threshold voltage.

a. Connect the ESP-2 to a voltage pulser (at the negative input) and set for 10 -mv output at 20,000 counts per minute. 
b. Turn speaker on. Set speaker rate to 1 (inside side access).

c. Set PHA-GROSS switch to GROSS.

d. Set THLD control (inside side access) fully clockwise (CW) to minimum sensitivity.

e. Adjust GAIN control (inside side cover) for the crossover point. The crossover point is the point at which the speaker rate just starts to increase and the display shows $5.00+01(5.0 \times 101)$ to $1.50+02\left(1.5 \times 10^{2}\right)$ counts per minute (cpm).

f. Set PHA-GROSS switch to PHA.

g. Set pulser output to $12 \mathrm{mv}$.

h. Adjust window potentiometer (through hole in case bottom) for crossover point.

i. Set pulse output to $5.6 \mathrm{mv}$.

j. Set PHA-GROSS switch to GROSS.

k. Adjust THLD control for crossover point.

1. Set PHA-GROSS switch to PHA.

$\mathrm{m}$. Verify a $50 \%$ window by slowly increasing pulser amplitude from 4 to $100 \mathrm{mv}$. Note the pulser amplitude as the clicks from the speaker start (Vl) and as it stops $(\mathrm{Vu})$. The window percent is calculated as indicated in the equation above.

n. If needed, readjust window potentiometer by repeating the steps above in order to obtain a $50 \%$ window.

\section{ESP-2 Analyzer Setup}

The following sets and stores the detector identification, calibration constant, rate count mode, and units of the count mode in the ESP-2 analyzer.

1. Turn the instrument on and push the "MODE" and "RESET" buttons simultaneously to get to the menu select mode. Depress the "-" button to select the parameter mode and to answer no to any question. The "+" key is for yes response to any question.

2. Push the "+" or "INC" button to insert the detector information. Release the button when the letter you desire appears. Then move the cursor to the right by pushing the "-" button. The maximum length of a description is 13 characters. The description should include detector identifying number and energy level (FID depress "RESET" key.

3. Next select the rate meter mode by pushing the "+" key.

4. Select the units of counts per minute (counts $/ \mathrm{min}$ ). 
5. Set Calibration constant $(C C)=1.00+00(1.0 \times 100)$.

6. Dead time should be set at $1.40-0.5\left(1.4 \times 10^{-5}\right) \mathrm{sec}$.

\section{Determining the Operating Voltage of the FIDLER Detector}

The ESP-2 analyzer has been calibrated but the operating voltage now needs to be selected for the radionuclide of interest. The purpose of the following steps is to determine the operating voltage range of the detector for Am-241. When determining the operating voltage, the detector voltage needs to be displayed on the LCD of the analyzer. Remernbe:, when adjusting or changing the high voltage, it is very difficult to set at exact voltage increments so set the high voltage as close to the desired value as possible.

1. Place the $\sim 10 \mu \mathrm{Ci}$ Am-241 check source directly under the FIDLER which is supported in the FIDLER stand (1-ft distance from detector face to source). Make certain the detector face is uncovered.

2. Select the "menu" mode by depressing the "MODE" and "RESET" keys simultaneously.

3. Select the "-" key for the ratemeter mode and respond with successive "+" keys until the high voltage (HV) is displayed.

4. Set the PHA-GROSS switch to PHA.

5. Set the HV to $5.99+02\left(5.99 \times 10^{2}\right)$ and take count-rate reading (in counts per minute) and record the count rate and actual voltage. The automatic-store mode is not convenient for this particular use as the parameters can not be displayed when auto store is used.

6. Next, increase the high voltage (HV) by about 50 volts and take a count rate reading. Record the count rate and voltage displayed.

7. Continue to increase the voltage by 50 volt increments, recording the count rate and voltage at each increment, to approximately 1500 volts.

8. Plot the voltage versus the count rate as in Figure 1. Note that the count rate will significantly increase and then decrease twice with increasing voltage.

From the plot, the correct operating voltage for Am-241 can be selected. The first peak (from 600 volts and up) on the plot is due to the $60 \mathrm{keV}$ gamma rays while the second peak is due to the $17 \mathrm{keV}$ x-rays, both energy peaks being associated with Am-241. Now that the correct operating voltages are known, the voltage for the 
detection of $60 \mathrm{keV}$ energy from Am-241 can be set and stored into the ESP-2 analyzer under "detector 1 ".

9. Turn the instrument on and then depress the "Mode" and "Reset" buttons simultaneously. Select "-/PARAMS" by depressing tne - vution. Push the "+" button to accept the other items until the high voltage ("HV", is indicated.

10. Set the high voltage for the voltage corresponding to the first peak of the plot. By simultaneously depressing the "Reset" and the "+" or "-" button, the high voltage may be increased or decreased, respectively.

\section{Procedure 2 - Determination of FIDLER Counting Efficiency and Angular Response Measurements}

This procedure determines the counting efficiency and measures the angular response of a FIDLER to a specific radionuclide source. The FIDLER counting efficiency should be checked prior to field measurements any time the FIDLER detector system has been calibrated. The FIDLER counting efficiency and angular response measurements are used in a computer code for quantifying soil radionuclide activity on the basis of detector geometries and radionuclide distributions in the soil. The computer code will be further discussed in the third BECAMP Quality Assurance DP (BECAMP-DP-403) and by Gouveia (1991). The computer code is discussed in detail by Anspaugh et al. (1972).

\section{A. FIDLER Counting Efficiency Test}

Now that the optimal operating voltage has been determined for the $60-\mathrm{keV}$ peak of Am-241, an efficiency check of the FIDLER should be conducted for verification of the voltage calibration. The FIDLER counting efficiency, $N_{0} / \Phi$, is the number of photopeak counts recorded by the FIDLER per photon per $\mathrm{cm}^{2}$ of detector face crosssection area when a point source is placed directly under the detector. For the operating voltage of $60 \mathrm{keV}$, a verified and traceable Am-241 check source $(\sim 10 \mu \mathrm{Ci})$ will be used. The FIDLER Calibration Data and Sign-Off Log (Attachment 1) will be used to record the data. The actual number of readings $(n)$ and the duration $(t)$ of the measurement will be determined by the Principal Investigator and documented in the monitoring protocol.

Equipment:

Eberline ESP-2 Smart Portable Analyzer

Bicron FIDLER probe

Am-241 check source (verified source of approximately $10 \mu \mathrm{Ci}$ )

FIDLER Stand 
1. Place the FIDLER in the FIDLER Stand so that the detector is directly one foot $(30.5 \mathrm{~cm})$ above the spot where the check source is to be placed.

2. Take $n$ readings for duration $t$ of background activity and record the countsper-minute (cpm) for each measurement.

3. Place the Am-241 check source, approximately $10 \mu \mathrm{Ci}$, one foot directly below the center of the detector face. Record the strength of the source and date determined.

4. Take $n$ readings for duration $t$ of the check source activity and record the counts-per-minute.

5. Calculate the average background activity and average check-source activity.

6. Subtract the average background activity from the average check source activity to get the net average check-source activity $\left(\mathrm{N}_{\mathrm{O}}\right)$.

7. Use the average check-source activity, No (in counts per minute), to calculate counting efficiency $\left(\mathrm{N}_{\mathrm{O}} / \Phi\right)$ in the following equation

$N_{0} / \Phi=N_{0} * 4 \pi * r^{2} /\left(s p * 2.22 \times 10^{6} * B i\right)$,

where $r$ is the distance from the source to the detector face (in $\mathrm{cm}$ ), $\mathrm{sp}$ is the radioactive strength (corrected for decay from date of calibration) of the check source (in $\mu \mathrm{Ci}$ ), and $\mathrm{Bi}$ is the branching intensity of the source. For this application, with $\mathrm{r}=30.5 \mathrm{~cm}$ and $\mathrm{Bi}=0.363$ for Am-241, Equation 1 can be simplified to

$$
\mathrm{No} / \Phi=\text { No } * 0.014 / \mathrm{sp} \text {. }
$$

8. Record the counting efficiency data on the FIDLER Calibration Data and Sign-Off Log.

The FIDLER counting efficiency should be equal to at least $80 \%$ of the surface area of the detector face. If the counting efficiency is less than that, readjust the HV setting with an Am-241 check source to find the optimum voltage for $60 \mathrm{keV}$ (Section D of Procedure 1). Repeat the determination of the counting-efficiency (Section A of Procedure 2). If the counting efficiency is still below $80 \%$ efficiency, repeat the procedure for setting a $50 \%$ window. Repeat the counting efficiency check. Continue this procedure until an acceptable counting efficiency is achieved. 


\section{B. FIDLER Angular-Response Measurements}

The following procedure should be conducted after the FIDLER and analyzer have been calibrated as described in Procedure 1 and passed the counting efficiency check described above. Record the angular-response measurements on the FIDLER Calibration Data and Sign-Off Log (Attachment 1 ). The actual number of readings ( $n$ ) and the duration $(t)$ of the measurement will be determined by the Principal Investigator and documented in the monitoring protocol.

\section{Equipment:}

Eberline ESP-2 Smart Portable Analyzer

Bicron FIDLER probe

Am-241 point source (verified source of approximately $100 \mu \mathrm{Ci}$ )

FIDLER Calibration Stand

Calibration Rig

1. Place the FIDLER in the calibration stand so that the face of the FIDLER is one meter from the base and arms of the calibration rig.

2. Turn on the power to the motor driving the calibration rig. Supply just enough power to the motor so not to overload the voltage equipment of the motor.

3. Take $n$ readings for duration $t$ of background activity. Record the five measurements.

4. Place the Am-241 point source (approximately $100 \mu \mathrm{Ci}$ ) at the base of the calibration rig directly below the detector.

5. With the calibration rig turning, take $n$ readings for duration $t$ with the source directly below the detector (referred to as the 0 degree position). Record the measurements.

6. Move the Am-241 source to the 15-degree position on the rig. With the calibration rig turning, and take $n$ readings for duration $t$ and record the measurements.

7. Continue taking $n$ readings for duration $t$ at 15 degree intervals along the calibration rig $(30,45,60,75$, and 90 degrees) and record the measurements.

8. Record the angular response data on the FIDLER Calibration Data and SignOff Log. 
The data collected from the measurement of FIDLER angular response will be used with the in situ measurements to quantify soil radionuclide concentrations. The procedures for taking in situ measurements are outlined in BECAMP DP-402 and the procedure for calculating radionuclide concentrations in soil from FIDLER in situ measurements can be found in BECAMP DP-403.

\section{REFERENCES}

Anspaugh, L.R., P.L. Phelps, G.W. Huckabay, P.H. Gudikesen, and C.L. Lindeken. 1972. Methods for the In Situ Measurement of Radionuclides in Soil. Lawrence Livermore National Laboratory, Livermore, CA. UCID-21573.

Essington, E. 1989. Quality Assurance Plan for the Basic Environmental Compliance and Monitoring Program (BECAMP). Los Alamos National Laboratory, Los Alamos, NM, LA-11652-MS.

Gouveia, F. 1991. Using FIDCAL for BECAMP. Lawrence Livermore National Laboratory, Livermore, CA. UCRL-MA-1049878 DRAFT.

Patton, S. 1990. Basic Environmental Compliance and Monitoring Program (BECAMP) FY 1990 Annual Work Plan. Livermore National Laboratory, Livermore, CA. UCAR-10220-90. 


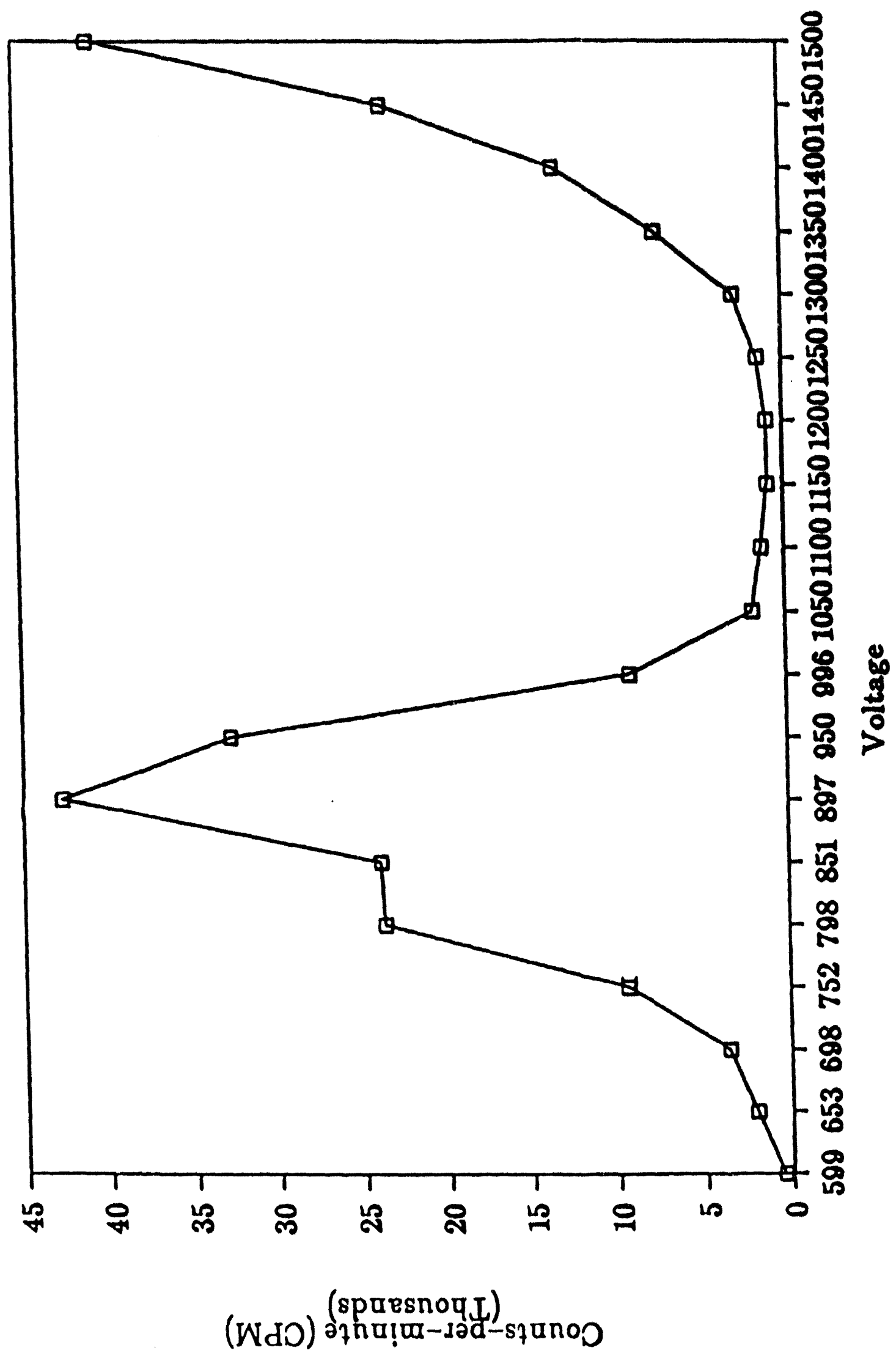


DETECTOR:

ANALYZER :

CORO LEGNTH:
FIOLER HEIGHT:

COUNT TIME 8
OUNTING EFFICIENCY

OURCE :

O-KOV BKG.

tVG. CPH
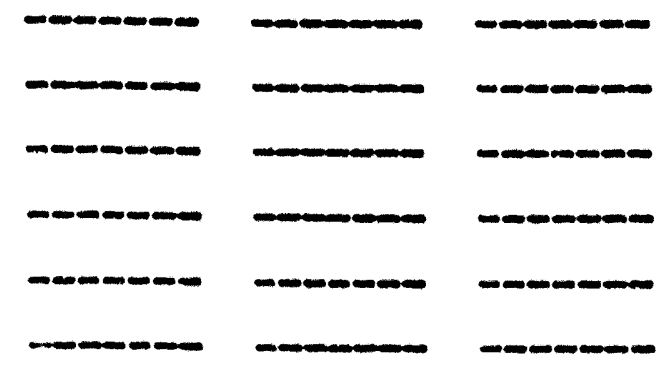

$-\infty-\infty=0$

-

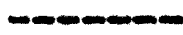

-

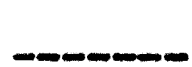

$60 \mathrm{KeV}$

:FFICIENCY:

ANGULAR RESPONSE MEASUREMENTS SOUREE:

$60^{\circ} \mathrm{KeV} \quad \mathrm{BKC}$
NAME:

OATE:

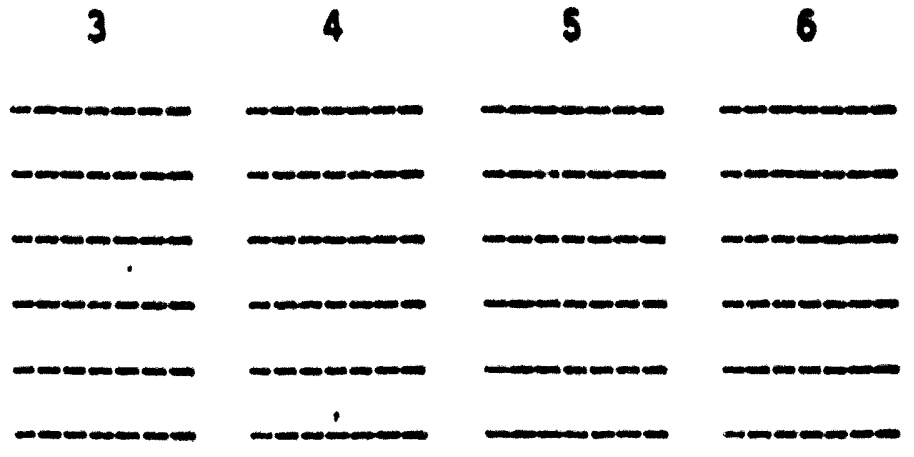

NAME 8

DATE\&

$\begin{array}{lllll}300 & 450 & 60^{\circ} & 750 & 900\end{array}$

$150 \quad 30^{\circ}$

$\square=$

$\longrightarrow$

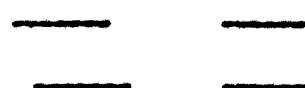

10

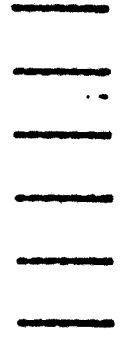

AVG. CPM

CHAIN OF CUSTODY

VAME:

نं̄itè

STGNATURE:

NAME:

DATE:

SI.GNATURE :

NAME :

$21 \div::$

SIGNATURE: 


\title{
BECAMP Quality Assurance Detailed Procedure for FIDLER In-Situ Measurements
}

\author{
Prepared by \\ Scott E. Patton \\ Environmental Sciences Division \\ Lawrence Livermore National Laboratory
}

May 1990

Prepared for the U.S. Department of Energy, Nevada Operations Office, sponsored Basic Environmental Compliance and Monitoring Program 


\section{BECAMP DETAILED PROCEDURE APPROVAL}

Effective Date_April 27, 1992

TASK NO_ 1 DP IDENTIFIER BECAMP-DP-402

PRINCIPAL INVESTIGATOR_Joseph H. Shinn

ORGANIZATION Lawrence Livermore National Laboratory

TITLE: BECAMP Quality Assurance Detailed Procedure for FIDLER In-Situ Measurements

\section{SIGNATURES:}

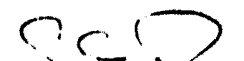

Sr: suinin

Preparer: S.E. Patton

7

kighe tricken

-Technicalkeviewer: J.H. Shinn

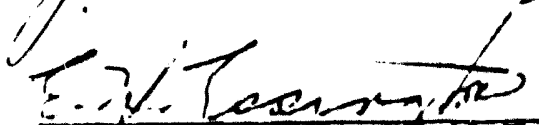

Quality Assurance: 4 . H. Essington

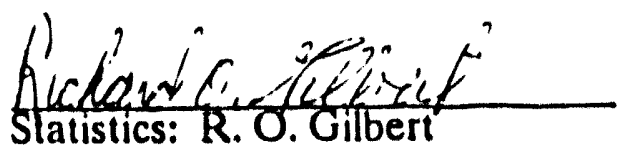

$\frac{7,12-1, c_{12}}{\text { Date }}$

$\frac{\text { Die }}{\text { Date }}$

$\frac{19 / 4192}{\text { Date }}$

Cesetic, 1497

COPIES TO:

J. B. Hall, Chief, Technology Development Brar.ch US Department of Energy, NVO

April 27, 1992

Date Sent

L. R. Anspaugh, BECAMP Scientific Director Lawrence Livermore National Laboratory

April 27, 1992

Däte Sent 


\section{PURPOSE}

The following procedure is to be used for making in situ measurements of americium-241 (Am-241) activity in soils with the FIDLER (field instrument for the detection of low-energy radiation) detector system. This procedure is the second of three BECAMP (Basic Environmental Compliance and Monitoring Program) Quality Assurance Detailed Procedures (DPs) for quantifying radionuclide concentrations in soils from in situ measurements with a FIDLER. The first DP is the procedure for the setup and calibration of the FIDLER and analyzer prior to taking in situ measurements and the third DP is the procedure for quantifying soil radionuclide concentrations from in situ FIDLER measurements of radionuclide activity in soil. This DP for in situ measurements with the FIDLER provides procedures for taking background and check source activity readings, making in situ measurements of Am-241 activity, and data collection and recording.

This detailed procedure fulfills a requirement of the BECAMP Quality Assurance Plan (Essington 1989). Detailed procedures define technical requirements, constraints, and procedural steps of scientific activities that are to be conducted by BECAMP Investigators. The DP will be reviewed for appropriate quality-assurance and statistical considerations and must be approved by BECAMP authorities as designated in the Quality Assurance Plar.

\section{SCOPE}

The procedures described in this BECAMP Quality Assurance DP are to be used for taking in situ measurements with a FIDLER detector system (a FIDLER scintillation detector and pulse-rate analyzer) for the determination of americium-241 activity in soils of the NTS (In this document and unless otherwise stated, FIDLER will refer to the FIDLER detector system comprised of a FIDLER scintillation detector and accomf anying pulse-rate analyzer). These procedures are to be used as a general guide for taking in situ measurements with the FIDLER. The field monitoring protocol provided by the BECAMP Principal Investigator will add specifics not covered in this DP or variations to this DP. (e.g., sampling locations, sampling frequency, and data collection).

This BECAMP QA Detailed Procedure provides the procedures for taking in situ measurements with the Eberline ESP-2 Smart Portable radiation-survey instrument in conjunction with the Bicron FIDLER scintillation detector. The ESP- 2 has many options and can be relatively complex to operate. However, due to its computerized nature it can also be extremely easy to use in the automatic inquiry mode. It is not the intent of this DP to provide an operating manual on the ESP-2 instrument. The use and operation of the ESP-2 can be found in "Model ESP-2 Eberline Smart Portable Technical Manual," December 1986, from Eberline. The ESP-2 operating manual can be found with the ESP-2 and FIDLER in the carrying case. 


\section{DEFINITIONS}

Analyzer - a pulse-rate meter that converts signals from a radiation-detection probe; specifically an Eberline ESP-2 Smart Portable Survey instrument with pulse-height analyzer (PHA) board.

FIDLER detector - a scintillation probe for the detection of low-energy radiation; specifically a Bicron probe, Model G5 "FIDLER" containing a 5-inch by 0.063 -inch Nal crystal.

FIDLER Stand - a metal tripod stand that supports the FIDLER probe $30 \mathrm{~cm}$ above the soil surface. The stand can be obtained from Reynolds Electric \& Engineering Company (REECo) Soils Laboratory, at the Nevada Test Site.

Source: Americium-241, $\sim 10 \mu \mathrm{Ci}$ check source

\section{RESPONSIBILITIES AND TRAINING}

The taking of in situ measurements with the FIDLER detector and ESP-2 analyzer is to be conducted by persons familiar with the ESP-2 analvzE: . In the current structure of the BECAMP program at the NTS (Patton 1990), the KEECo Soils Laboratory will have the responsibility of taking in situ measurements with the FIDLER. The personnel of the Soils Laboratory will be familiar with the ESP-2 analyzer prior to conducting field work. Prior to taking in situ measurements, a demonstration of proficiency in operation of the ESP-2/FIDLER will be demonstrated to the Principal Investigator requesting the measurements.

This procedure is to be conducted by persons familiar with the ESP-2 analyzer. Those persons involved with taking in situ FIDLER measurements will have working knowledge of these procedures. The responsibility of training belongs to the Principal Investigator of BECAMP Task 1 (currently Joseph $H$. Shinn of Lawrence Livermore National Laboratory or the Principal Investigator's designee).

\section{RECORD KEEPING}

A BECAMP FIDLER Measurements form has been prepared (Attachment 1) and will be used for collection of data in the field. The field data will also be electronically stored by the ESP-2 and transferred to a computer in the REECo Soils Laboratory. The electronic copy will be printed and verified by comparison with the Data Collection form. Both the written form and the magnetic form of the data will be saved. Quality Assurance sign-offs are found on the BECAMP FIDLER Measurements form. A copy of the data collection form, electronic media, and a printed copy of the data on the electronic media will be sent to the Principal Investigator upon completion of the investigation. 


\section{PROCEDURES}

The following is a general procedure for taking in situ measurements with the FIDLER. Greater detail or monitoring specifics will be found in the field monitoring protocol supplied by the Principal Investigator. Before proceeding to the field, the FIDLER should be calibrated per BECAMP-DP-401.

\section{A. Background Activity and Check Source Measurements}

Measurements of background activity and source checks should to be made in several places prior to entering the survey area. The Principal Investigator will provide the locations for the measurement of background activity and source checks, the number of measurements $(n)$, and the duration of each measurement $(t)$ in the fieldmonitoring protocol. Measurements should be made prior to field monitoring and upon completion of the monitoring. Measurements should also be made whenever leaving or entering the monitoring area or when interrupting a series of field measurements (e.g., lunch, breaks), or as directed by the field-monitoring protocol.

a. At each site, take $n$ measurements of $t$ duration with the FIDLER detector in the FIDLER stand (make certain the cover is removed from the face of the detector). record the counts-per-minute of each measurement on the BECAMP FIDLE? Measurements form and store the reading in the memory of the ESP-2 analyzer.

b. At each site, take $n$ measurements readings with the $\sim 10 \mu \mathrm{Ci}$ Am-241 check source directly below (on soil surface) the face of the FIDLER detector (which is in the FIDLER stand). Record and store the counts-per-minute readings.

\section{B. Field Measurements}

The field monitoring protocol submitted by the Principal Investigator will provide the specifics for monitoring with the FIDLER including the monitoring location, number of measurements $(n)$, and duration of measurement $(t)$. The following is a general procedure for in situ measurements with the FIDLER.

a. At each monitoring location, take $n$ measurements of $t$ duration with the FIDLER detector in the FIDLER stand. Record the counts-per-minute of each measurement on the BECAMP FIDLER Nieasurements form and store the reading in the memory of the ESP-2 analyzer.

b. The location of the FIDLER with respect to the sampling location (marker) will be noted on the data sheet.

c. The description of the monitoring location, per the key on the BECAMP FIDLER Measurements form, will be noted on the form. 


\section{REFERENCES}

Essington, E. 1989. Quality Assurance Plan for the Basic Environmental Compliance and Monitoring Program (BECAMP). Los Alamos National Laboratory, Los Alamos, NM, LA-11652-MS.

Patton, S. 1990. Basic Environmental Compliance and Monitoring Program (BECAMP) FY 1990 Annual Work Plan. Lawrence Livermore National Laboratory, Livermore, CA. UCAR-10220-90. 


\section{ECAMP FIDLER MEASUREMENTS}

YOJEf?:

HTE :

¿ELQ CREW:

-

JUNT TIME:

HECK SOURCE:

ETECTOR HEIGITT:
DESC. OF LOCATION:

1. DESERT PAVEMENT

2. BLOW-SAND MOUND

3. BETWEEN BUSHES

4. SAND \& GRAVEL

5. WASH AREA

6. RODENT BURROWS
INSTRUMENT:

EFFICIENCY:

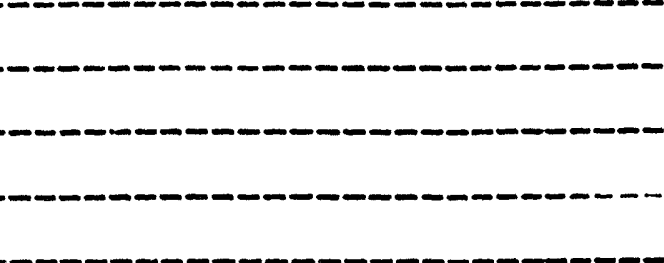

\section{FIDLER MEASUREMENTS}

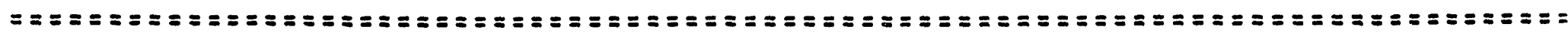
OCATION

DESC. OF

COUNTS

NET-CPM

COMMENTS

LOCATION (CPM)

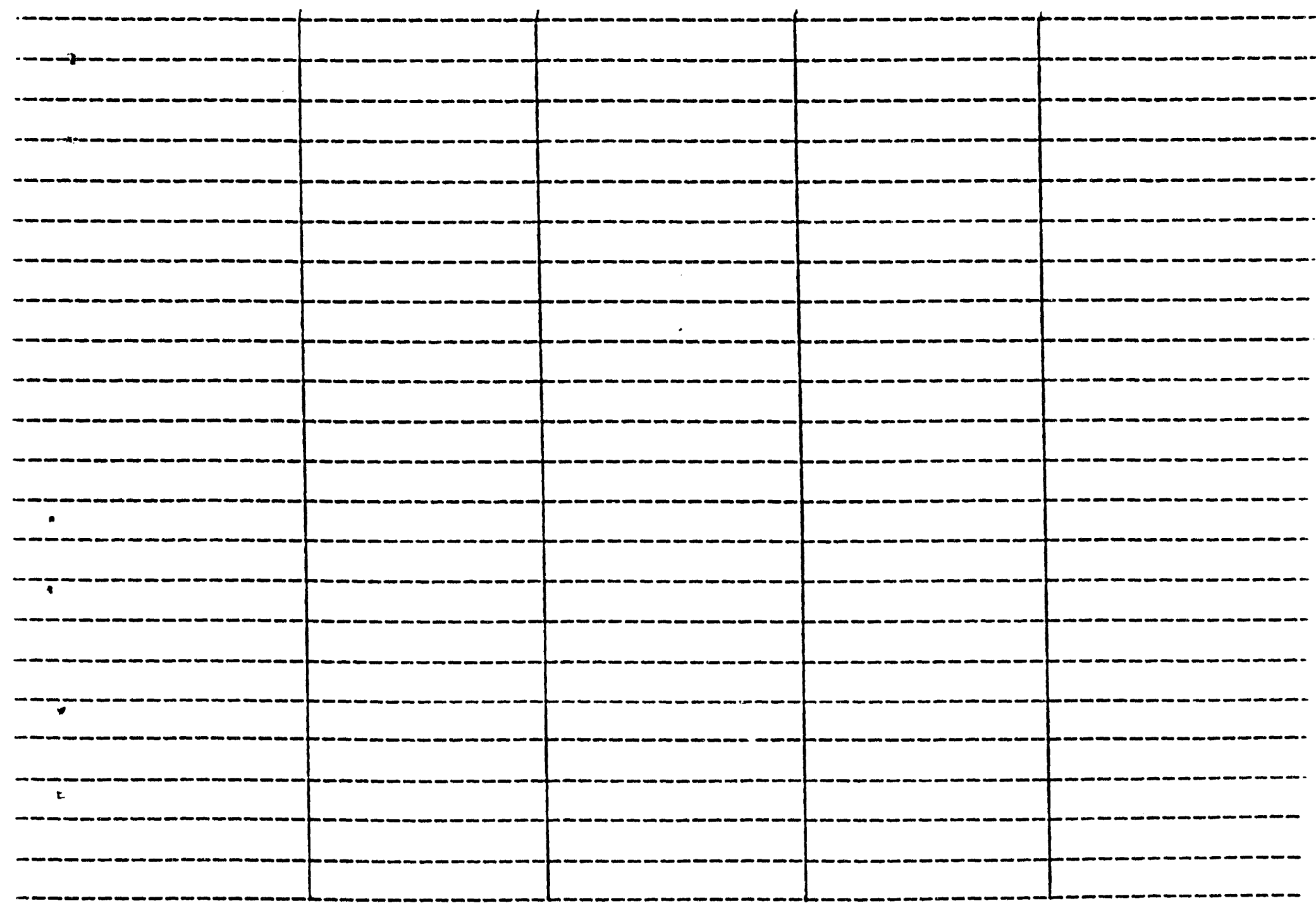




\title{
BECAMP Quality Assurance Detailed Procedure for FIDLER Data Analysis
}

\author{
Prepared by \\ Scott E. Patton \\ Environmental Sciences Division \\ Lawrence Livermore National Laboratory
}

May 1991

Prepared for the U.S. Department of Energy, Nevada Operations Office, sponsored Basic Environmental Compliance and Monitoring Program 


\section{BECAMP DETAILED PROCEDURE APPROVAL}

Effective Date April 27, 1992

TASK NO 1 DP IDENTIFIER BECAMP-DP-403

PRINCIPAL INVESTIGATOR Joseph H. Shinn

TITLE: BECAMP Quality Assurance Detailed Procedure for FIOLER Data Analys is

SIGNATURES:

Sc D

Preparer: S.E. Patton

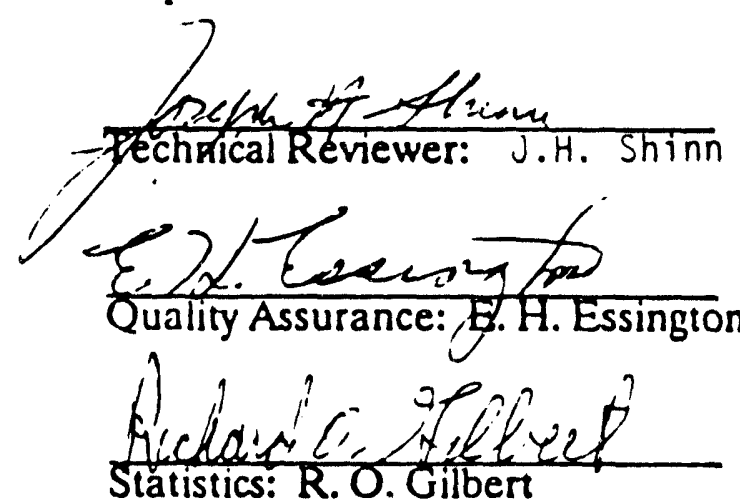

cer cetsiz Date

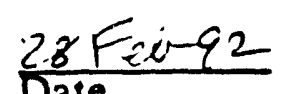

Date

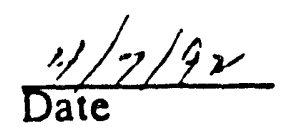

Cosel 10,1093

COPIES TO:

J. B. Hall, Chief, Technology Development Branch US Department of Energy, NVO

April 27, 1992

Date Sent

Apri] 27, 1992

L. R. Anspaugh, BECAMP Scientific Director Lawrence Livermore National Laboratory

Date Sent 


\section{PURPOSE}

The following procedure is to be used in quantifying soil radionuclide concentrations from in situ measurements with a FIDLER (field instrument for the detection of low-energy radiation) detector system. Specific instructions on data handling and analysis or variations on this procedure will be stated in the Monitoring Plan designed by the Principal Investigator for a particular monitoring exercise. This procedure (BECAMP-DP-403) is the third of three Basic Environmental Compliance and Monitoring Program (BECAMP) Quality Assurance (QA) Detailed Procedures for using a FIDLER for in situ measurements. The first QA Detailed Procedure (BECAMPDP-401) is the procedure for the setup and calibration of the FIDLER and the second procedure (BECAMP-DP-402) is for taking in situ measurements with the FIDLER.

This detailed procedure fulfills a requirement of the BECAMP Quality Assurance Plan (Essington 1989). Detailed procedures define technical requirements, constraints, and procedural steps of scientific activities that are to be conducted by BECAMP Investigators. The DP must be reviewed for appropriate quality-assurance and statistical considerations and must be approved by BECAMP authorities as designated in the Quality Assurance Plan.

\section{SCOPE}

The procedures described in this BECAMP QA Detailed Procedure are to be used in analyzing the data from the calibration of the FIDLER and the data from field measurements with the FIDLER in order to quantify americium-241 (Am-241) and plutonium-239,240 (Pu-239,240) activity in soils of the Nevada Test Site (NTS) and its environs. The main component of this DP is a user-interface computer program called FIDCAL (Gouveia 1991) that will provide factors based on the FIDLER counting efficiency and angular response measurements (BECAMP-DP-401), when multiplied by FIDLER in situ measurements (BECAMP-DP-402), will give soil radionuclide concentrations (in picocuries per square centimeter $\left(\mathrm{pCi} / \mathrm{cm}^{2}\right)$ ).

The FIDCAL program provides a user interface to a program named FLUX (Anspaugh et al. 1972 and Anspaugh 1976) that computes the angular response of the detector and the flux of photons incident on the detector per unit soil radioactivity. FIDCAL also incorporates calculations developed by Beck et al. (1964) that relates to the vertical distribution of radioactivity in soil. The input to FIDCAL includes the data from the detector angular response measurements, the vertical distribution of the radionuclide in soil, mass-attenuation coefficients of soil and air, and soil density. The data from the detector angular response measurements is obtained from the calibration of the detector (BECAMP-DP-401) and the remaining inputs are supplied by the Principal Investigato: in the monitoring plan. The output from FIDCAL are three factors; 
$N_{0} / \Phi$, the counting efficiency of the detector,

$N_{f} / N_{0}$, the angular response of the detector, and

$\Phi / S$, the flux of gamma rays striking the detector.

The three factors from above are used in the following equation to quantify soil radionuclide concentrations;

$$
A=G /(B i * 2.22 * t),
$$

where
$A=$ concentration of Am-241 in soil ( $\left.\mathrm{pCi} / \mathrm{cm}^{2}\right)$,
$G=$ gamma flux per $\mathrm{cm}^{2}$ of soil $\left(\gamma / \mathrm{cm}^{2}\right)$,
$\mathrm{Bi}=$ the branching intensity of Am-241; the number of gamma photons per disintegration $(\gamma / d)$,
$2.22=$ the conversion of disintegrations per minute per $\mathrm{pCi}(\mathrm{d} / \mathrm{min} / \mathrm{pCi})$, and
$t=$ measurement counting time $(\min )$.

In Equation 1

$$
G=N_{f} / X,
$$

where

$$
\begin{aligned}
& N_{f}=\text { the net counts (counts-per-minute) from in situ measurements, and } \\
& X=\left(N_{0} / \Phi * N_{f} / N_{0} * \Phi / S\right)
\end{aligned}
$$

The FIDCAL document (Gouveia 1991) uses Equation 1 to convert values of net Am-241 counts to the flux of gamma rays emitting from the soil surface, and then to the concentrations of Am-241 and Pu-239,240 in the soil column. To get the Pu-239,240 activity (P) from the Am-241 activity (A), FIDCAL uses a Pu/Am ratio (R) in

$$
P=A * R
$$

The Pu/Am ratio in soils is site specific and the ratio will be supplied by the Principal Investigator in the monitoring plan.

It is not the intent of this QA Detailed Procedure to describe in detail the content of FIDCAL or the calculations used to determine radionuclide concentrations from in situ measurements of soil activity. This information can be found in the papers and documents referenced in this DP. In addition, this DP does not provide instructions for the operation of a computer or associated equipment. It is the responsibility of the user of FIDCAL to be competent in the use and operation of the computer running FIDCAL. 


\section{DEFINITIONS}

I

The outputs from FIDCAL will include factors used in computing soil radionuclide concentrations $\left(N_{0} / \Phi, N_{f} / N_{0}\right.$, and $\left.\Phi / S\right)$, the values for the site specific parameters used in the computations (soil density and mass-attenuation coefficients of soil and air), and a plot of the angle of source verses detector response. The outputs from FIDCAL and the spreadsheet used in quantifying radionuclide activity from in situ measurements will be added to the input data sheets and submitted to the Principal Investigator upon completion of the analysis. 


\section{PROCEDURE FOR DATA ANALYSIS}

The main component in ite analysis of in situ FIDLER measurements is using the FIDCAL program (Gouveia 1991) to provide the factors $\left(N_{0} / \Phi, N_{f} / N_{0}\right.$, and $\Phi / S$ ) necessary to convert in situ measurements to soil radionuclide concentrations as given in Equation 1:

$$
A=G /(B i * 2.22 * t) .
$$

For $\mathrm{Am}-241$ at $60 \mathrm{keV}, \mathrm{Bi}=0.363$. Equation 1 then can be rewritten as

$$
A=G * 1.24 / t
$$

Substituting Equation 2 in Equation 4 gives

$$
A=N_{f} * 1.24 /(X * t) \text {. }
$$

It is recommended that a spreadsheet be constructed, by hand or on a computer, that will use the net average in situ measurements $\left(N_{f}\right)$ for each FIDLER sample site (see BECAMI'- Li $^{2}-402$ ) and the factors from FIDCAL. To get Pu-239,240 activity (P), multiply the Am-241 activity (A) by the Pu/Am ratio (R) as in Equation 3.

For example, construct a spreadsheet using the rows for each in situ sample site and columns with the following headings and values.

COLUMN
1
2
3
4
5

$$
\begin{gathered}
\text { HEADING } \\
\text { Field Measurements } \\
X \\
\text { Gamma flux } \\
\text { Am-241 activity } \\
\text { Pu-239,240 activity }
\end{gathered}
$$

Then,

Column $3=$ Column $1 /$ Column 2, i. e., $G=N_{f} / X$,

Column $4=$ Column $3 * 1.24 / t$, i. e., $A=G * 1.24 / t$,

Column $5=$ Column $4 * R$, i. e., $P=A * R$.

The spreadsheet will be forwarded to the Principal Investigator as well as a copy of the FIDCAL output of the three factors and the FIDCAL plot of the detector angular response. Included with the spreadsheet will be the calibration data form (from BECAMP-DP-401) and field data sheet and notes (from BECAMP-DP-402). 


\section{REFERENCES}

Anspaugh, L.R., P.L. Phelps, G.W. Huckabay, P.H. Gudikesen, and C.L. Lindeken. 1972. Methods For The In Situ Measurement Of Radionuclides In Soil. Lawrence Livermore National Laboratory, Livermore, CA. UCID-21573.

Anspaugh, L.R. 1976. "In-situ Methods for Quantifying Specific Radionuclides," IEEE Transaction on Nuclear Science NS -23, 1190-1196.

- Beck, H.L., W.J. Condon, and W.M. Lowder. 1964. Spectrometric Techniques for Measuring Environmental Gamma Radiation. USAEC, Health and Safety Laboratory, New York, Rept. HASL-150.

Essington, E. 1989. Quality Assurance Plan for the Basic Enoironmental Compliance and Monitoring Program (BECAMP). Los Alamos National Laboratory, Los Alamos, NM, LA-11652-MS.

Gouveia, F. 1991. Using FIDCAL for BECAMP. Lawrence Livermore National Laboratory, Livermore, CA. UCRL-MA-1049878 DRAFT.

- Patton, S. : 91 . Basic Environmental Compliance and Monitoring Program (BECAMP) FY 1991 Annual Work Plan. Lawrence Livermore National Laboratory, Livermore, CA. UCAR-10:20-91. 

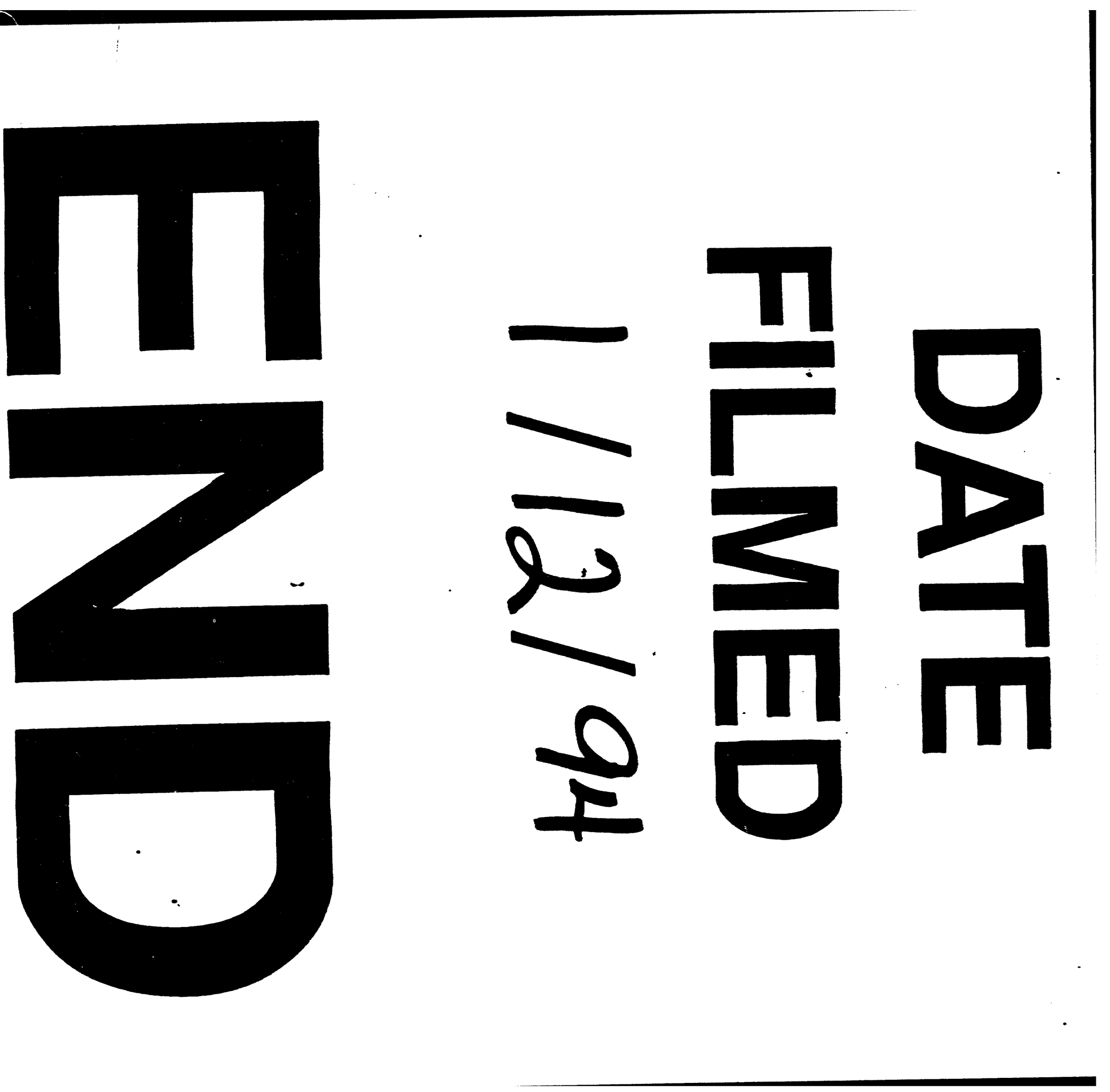


$$
\text { 10 }
$$
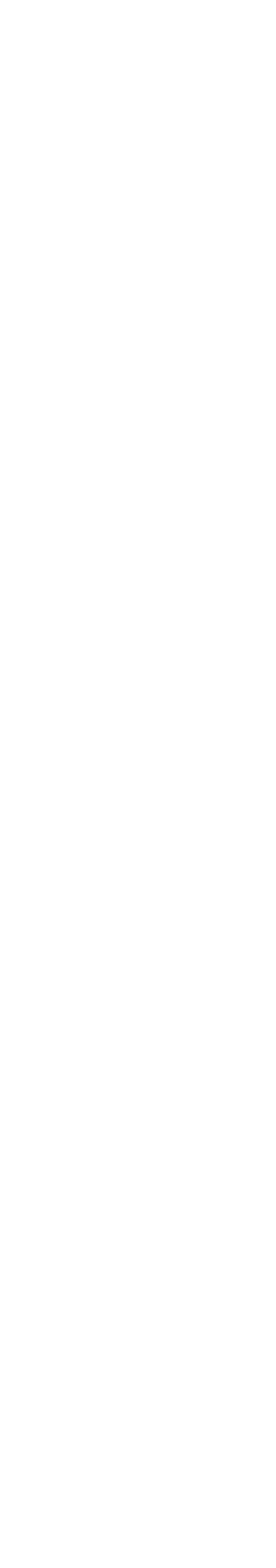